\title{
Interpolating between multi-center microstate geometries
}

\author{
Masaki Shigemori \\ Department of Physics, Nagoya University, \\ Furo-cho, Chikusa-ku, Nagoya 464-8602, Japan \\ Center for Gravitational Physics, \\ Yukawa Institute for Theoretical Physics, Kyoto University, \\ Kitashirakawa Oiwakecho, Sakyo-ku, Kyoto 606-8502, Japan \\ E-mail: shige@yukawa.kyoto-u.ac.jp
}

ABSTRACT: We study interpolation between two multi-center microstate geometries in $4 \mathrm{~d} / 5 \mathrm{~d}$ that represent Lunin-Mathur geometries with circular profiles. The interpolating solution is a Lunin-Mathur geometry with a helical profile, and is represented by a 2center solution with a codimension-2 source. The interpolating 2-center solution exhibits interesting features such as some of the charges being delocalized, and some of the charges getting transferred from the codimension- 2 center to the other, codimension- 3 center as the interpolation proceeds. We also discuss the spectral flow of this entire process and speculate on the relevance of such solutions to understanding general microstates of 3charge black holes.

KeYwords: AdS-CFT Correspondence, Black Holes, Black Holes in String Theory

ArXiv EPrint: 2105.11639 


\section{Contents}

1 Introduction and summary 1

2 Setup 6

2.1 Harmonic solutions 6

2.2 Lunin-Mathur geometries 9

$\begin{array}{ll}2.3 & \text { Relation to harmonic solutions } \\ \end{array}$

3 Codimension-2 Lunin-Mathur solution 13

\begin{tabular}{ll}
3.1 & Building blocks \\
\hline
\end{tabular}

$\begin{array}{lll}3.2 & \text { Codimension-2 source } & 17\end{array}$

$\begin{array}{lll}3.3 & \text { Codimension-3 sources } & 18\end{array}$

3.4 Integrability and no-CTC conditions 20

$\begin{array}{lll}3.5 & \text { Comments } & 21\end{array}$

4 Deriving harmonic functions from 6d 22

5 Dual CFT perspective and spectral flow 23

5.1 Dual CFT states 23

5.2 Spectral flow 24

$\begin{array}{lll}5.3 & \text { Fractional spectral flow } & 27\end{array}$

6 Discussions $\quad 28$

$\begin{array}{lr}\text { A Duality transformation } & 29\end{array}$

$\begin{array}{ll}\text { B Coordinate systems } & 30\end{array}$

C Functions $H, \mathcal{I}_{m n}, \gamma \quad 31$

C.1 The harmonic function $H \quad 31$

C.2 The integrals $\mathcal{I}_{m n}$

C.3 The monodromic harmonic function $\gamma \quad 34$

$\begin{array}{lll}\text { C.4 Various relations } & 35\end{array}$

\section{Introduction and summary}

String theory contains various extended objects - branes - and allows configurations with multiple such branes bound together by their gravitational and gauge interactions. In supergravity, such configurations are realized by solutions with multiple centers representing branes. Among such multi-center solutions in supergravity, an important class 
of solutions is the one in four and five dimensional supergravity that is supersymmetric and characterized by harmonic functions in $\mathbb{R}^{3}[1-7]$. They have various applications such as the attractor mechanism [8-14], split attractor flows and wall crossing [3, 15-18], and microstate geometries $[6,19,20]$.

In this note we will call these solutions "harmonic solutions", because their construction heavily relies on harmonic functions. In most literature those harmonic solutions are assumed to have sources of codimension three, but they can also have codimension- 2 sources. ${ }^{1}$ Codimension-2 sources can be produced by the supertube transition [21] and harmonic functions will have non-trivial monodromies around a curve in $\mathbb{R}^{3}$. Some examples of codimension-2 harmonic solutions were studied in [22, 23].

In this note, we extend the examples of codimension- 2 harmonic solutions by studying the Lunin-Mathur geometries [24, 25] in the framework. The Lunin-Mathur geometries are smooth, horizonless solutions of type IIB supergravity in $\mathbb{R}_{t} \times \mathbb{R}^{4} \times S_{y}^{1} \times T^{4}$ and represent microstates of the D1-D5 2-charge system. Ignoring $T^{4}$ directions they can be regarded as solutions of $6 \mathrm{~d}$ supergravity. The geometries are parametrized by profile functions which describe the shape in $\mathbb{R}^{4}$ of the worldvolume of a Kaluza-Klein monopole (KKM) produced by the supertube transition of D1- and D5-branes. For some special choices of the profile functions, via duality transformations, the Lunin-Mathur geometries can be described by harmonic solutions with codimension-3 sources. Here we consider more general profile functions, for which the harmonic functions have codimension- 2 sources as well. ${ }^{2}$

Our purpose here is to develop techniques to construct harmonic solutions with codimension-2 sources. The harmonic solution involves harmonic functions commonly denoted by $\left(V, K^{I}, L_{I}, M\right)=: \mathcal{H}$ where $I=1,2,3$. The construction proceeds in layers, in that one constructs the harmonic functions in the order of $V, K^{I}, L_{I}, M$. In the previous work [22, 23], solutions with trivial $V$ (i.e. $V=1$ ) were studied. In the current note, we will extend this to solutions with non-trivial $V$. This is a step forward for constructing the most general codimension-2 harmonic solutions.

In the remainder of this section, we introduce the setup and summarize our main findings.

The Lunin-Mathur geometry is parametrized by profile functions $g_{m}(\lambda), m=1,2,3,4$ in $\mathbb{R}^{4}$, where $g_{m}(\lambda+L)=g_{m}(\lambda)$ with $L$ a constant. One of the simplest Lunin-Mathur geometry is given by the following profile:

$$
g_{1}(\lambda)+i g_{2}(\lambda)=a e^{i k \Omega \lambda}, \quad g_{3}(\lambda)+i g_{4}(\lambda)=0,
$$

where $a>0, k \in \mathbb{Z}_{>0}$ and $\Omega=2 \pi / L$. This is a circle in the $x_{1}-x_{2}$ plane (and a point at the origin in the $x^{3}-x^{4}$ plane). Using the Hopf fibration, we can project this $\mathbb{R}^{4}$ profile onto $\mathbb{R}^{3}$ in which the harmonic functions $\left(V, K^{I}, L_{I}, M\right)$ of the harmonic solution live. If we take the Hopf fiber direction $\psi$ to be the same as the circle direction of the profile (1.1), the

\footnotetext{
${ }^{1}$ Codimension-1 singularities are also possible, although we do not consider them in this note.

${ }^{2}$ The duality transformations at the supergravity level involve smearing, and therefore the profile function in $\mathbb{R}^{4}$ gets smeared along a direction $\psi$ along which T-duality is taken. This is unlike the formulation of [26] which generalizes the harmonic function to depend on $\psi$.
} 
harmonic functions are given by

$$
\begin{aligned}
& V=\frac{1}{r}, \quad K^{1}=K^{2}=0, \quad K^{3}=\frac{Q_{5} \Omega k}{2}\left(\frac{1}{\Sigma}-\frac{1}{r}\right), \\
& L_{1}=\frac{Q_{1}}{4 \Sigma}, \quad L_{2}=\frac{Q_{5}}{4 \Sigma}, \quad L_{3}=1, \quad M=\frac{Q_{5} \Omega k \widetilde{a}}{4 \Sigma} \text {. }
\end{aligned}
$$

Here the coordinates of $\mathbb{R}^{3}$ are $\mathbf{y}=\left(y_{i}\right), i=1,2,3$, and we defined $r \equiv|\mathbf{y}|, \Sigma \equiv|\mathbf{y}-\widetilde{\mathbf{a}}|$, $\widetilde{\mathbf{a}} \equiv(0,0,-\widetilde{a}), \widetilde{a}=a^{2} / 4$. These harmonic functions (1.2) have codimension-3 singularities at the origin $\mathbf{y}=0(r=0)$ and at a point on the negative $y_{3}$ axis, $\mathbf{y}=\widetilde{\mathbf{a}}(\Sigma=0)$. The singularities in $L_{1}, L_{2}$ at $\Sigma=0$ correspond to the D1 and D5-brane charges that we start with, while the singularity in $K^{3}$ at $\Sigma=0$ corresponds to the KKM charge of the D1-D5 supertube along the Hopf fiber direction $\psi$. To avoid possible confusion, in the following, we refer to this charge appearing as a codimension-3 source in $K^{3}$ as the $D_{4}$ charge, borrowing its dual type IIA interpretation. This solution has a U(1) rotational symmetry about the $y_{3}$ axis.

The need for the $1 / r$ term in $K^{3}$ is not so obvious from the viewpoint of the harmonic function, but this is what one gets from reducing the Lunin-Mathur solution. As we will see in the main text, this is necessary for the gauge field in the Lunin-Mathur solution, which involves $V^{-1} K^{3}$, to vanish at infinity. Alternatively, we can regard this $1 / r$ term as coming from the "gauge transformation" [27] of harmonic solutions, under which physical fields are invariant:

$$
V \rightarrow V, \quad K^{I} \rightarrow K^{I}+c^{I} V
$$

with $c^{I}$ arbitrary constants (here we are only showing the $V, K^{I}$ part; for the full expression including $L_{I}$ and $M$, see (2.6)). It is clear that by choosing $c^{3}$ appropriately we can change the coefficient of the $1 / r$ term in $K^{3}$ as we want.

A more general profile - the object of main interest in the current note - is

$$
g_{1}(\lambda)+i g_{2}(\lambda)=a e^{i k \Omega \lambda}, \quad g_{3}(\lambda)+i g_{4}(\lambda)=b e^{-i k^{\prime} \Omega \lambda},
$$

where $a, b \geq 0$ and $k, k^{\prime} \in \mathbb{Z}_{>0}$. This is like a helix, going in circles in both $x_{1}-x_{2}$ and $x_{3}-x_{4}$ directions, with pitches $k, k^{\prime}$. When $b=0$, this reduces to (1.1). For $b>0$, the $\psi$ direction is not an isometry of the Lunin-Mathur solution, but we can still reduce it to $\mathbb{R}^{3}$ after smearing it along the $\psi$ direction. The projected profile in $\mathbb{R}^{3}$ is like a latitude line on the globe. The explicit harmonic functions can be found in section 3. As we will see in the main text, the parameters $a, b$ are not arbitrary but constrained to satisfy

$$
a^{2} k^{2}+b^{2} k^{2}=\text { const }=\frac{Q_{1}}{Q_{5} \Omega^{2}} .
$$

Let's say we start with $a>0, b=0$, which corresponds to (1.1), and increase $b$ satisfying (1.5), finally ending with $a=0, b>0$. The projected profile in the $\mathbf{y}$ space starts as a point at the "south pole" of a spheroid (at $a>0, b=0$ ) and, as we increase $a$, becomes a latitude line. As we go up the spheroid from the south toward the north, the latitude line gets larger and then smaller, finally collapsing to a point at the "north pole" 


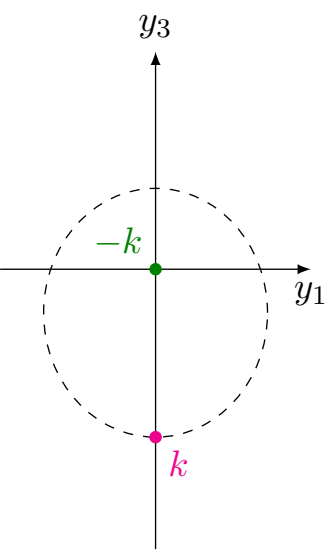

(a) south pole limit

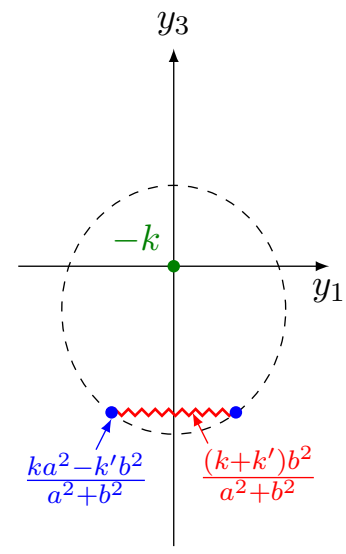

(b) "southern" case

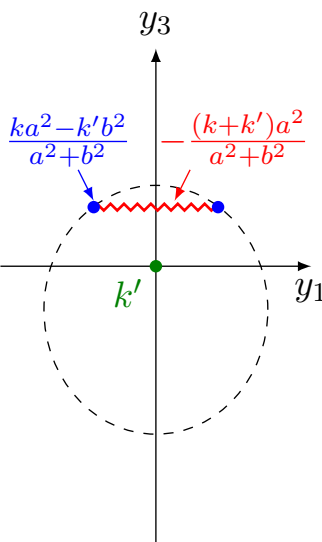

(c) "northern" case

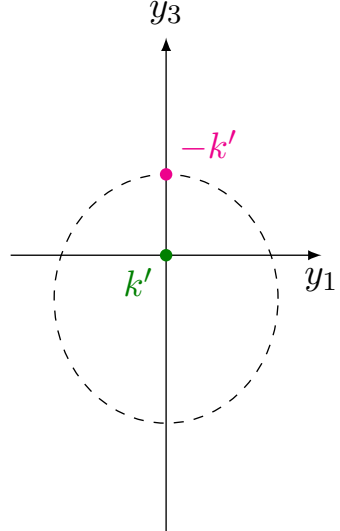

(d) north pole limit

Figure 1. The $\mathbb{R}^{3}$ profile and D4 charges of the "helical" solution, as we change the parameters $a, b$. (a): the south pole limit $(a>0, b=0)$. The profile is a point at the south pole of a spheroid (dashed ellipse). The purple dot represents the D4 charge at $\Sigma=0$, while the green dot the $r=0$ center. The D4 charge is shown next to each center in units of $Q_{5} \Omega / 2$. (b) as we make $b$ nonzero, the profile goes off the south pole and becomes a curve $\mathcal{C}$ along a latitude line of the spheroid. The blue dots represent the location of $\mathcal{C}$. The magenta zigzag line between them represents the branch cut coming from the multi-valuedness of $K^{3}$. Delocalized D4 charges are distributed on it. (c) As we increase $b$ and the profile $\mathcal{C}$ goes up, the branch cut crosses the $r=0$ center. Some of the delocalized D4 charge has been transferred to the $r=0$ center. (d) In the north pole limit $(a=0)$, the profile collapses to a point at the north pole. See the main text for more detail.

(at $a=0, b>0$ ); see the purple and blue dots in figure 1 . When $a, b>0$, the latitude line $\mathcal{C}$ is a codimension-2 singularity, around which $K^{3}$ has an additive a monodromy. This singularity represents the KKM charge of the D1-D5 supertube along $\mathcal{C}$. Let us refer to this charge appearing as a codimension-2 source in $K^{3}$ as the NS5 charge, borrowing its dual IIA interpretation. This is the most general Lunin-Mathur solution reduced to $4 \mathrm{~d}$ that preserves the $\mathrm{U}(1)$ rotational symmetry about the $y_{3}$ axis.

Even for $a, b>0$ we have the D4 charge (KKM charge along $\psi$ ), appearing as codimension-3 sources in $K^{3}$. It is interesting to see what happens to the D4 charge as we go from the south pole to the north pole. Being a dipole charge, the D4 charge is not conserved from the $5 \mathrm{~d}$ viewpoint, because the Hopf fiber that it is wrapping is contractible in $\mathbb{R}^{4}$. However, from the $4 \mathrm{~d}$ viewpoint, the D4 charge is an ordinary (monopole) charge as any other charges and is conserved. This is possible because the origin of $\mathbb{R}^{4}$, which is a D6-brane at $r=0$ in the IIA interpretation, can also carry D4 charges; as the profile moves on the spheroid, some of the D4 charge can be transferred to the D6 center at $r=0$, so that the total D4 charge is conserved. This is the same mechanism as in [28], where they studied how the charge of a fundamental string wrapping the special circle of a KKM background can be conserved, even though the circle is contractible.

Here we stop to note that there are multiple notions of charges [29] and the one we are talking about is the Page charge, which is conserved, localized, and quantized, but 
changes under large gauge transformations. For harmonic solutions, the D4 Page charge is measured by [22]

$$
-\frac{1}{4 \pi} \int_{M} *_{3} d K^{3}
$$

where $*_{3}$ is the Hodge star for flat $\mathbb{R}^{3}$ and $M$ is a Gaussian surface. If $K^{3}$ is single-valued, this simply picks up the coefficients of poles in $K^{3}$.

In the present setting, charge conservation works as follows. Because of the non-singlevaluedness of $K^{3}$, in addition to the charge source appearing as a pole in the harmonic function, there also are "delocalized" charges that can be measured by the Gaussian surface surrounding the disk whose boundary is $\mathcal{C} .^{3,4}$ This disk is the branch cut across which $K^{3}$ is discontinuous. As $\mathcal{C}$ moves up on the spheroid, when the center at $r=0$ crosses the branch cut, the delocalized charge gets transferred to the $r=0$ center. This process is described in figure 1. For $a>0, b=0$ (south pole), there is a non-vanishing D4 charge but no NS5 charge (figure 1(a)). As we move off the south pole, we start to have a non-vanishing NS5 charge along a now finite $\operatorname{ring} \mathcal{C}$. The $\mathrm{D} 4$ charge is divided into a localized part on curve $\mathcal{C}$ and a delocalized part distributed over the branch cut (figure 1(b)). After the branch cut has passed the $r=0$ center, the delocalized charge has changed by the amount transferred to the $r=0$ center (figure $1(\mathrm{c})$ ). Finally, when we reach the north pole, curve $\mathcal{C}$ shrinks to a point and we are left with a different amount of D4 charge (figure 1(d)).

So, the trivial-looking process of continuously changing the Lunin-Mathur profile in $6 \mathrm{~d}$ leads to a non-trivial process in the $4 \mathrm{~d}$ harmonic solution, in which the $\mathrm{D} 4$ charge gets transferred to the D6 center by way of the dimension-2 NS5-brane. The significance of this is further discussed in section 5 in relation to spectral flow in the dual CFT. Generalizations of such processes of charge transfer are expected to realize more general topology-changing processes that presumably play an important role in understanding the microscopic physics of black holes, as will be discussed in section 6 .

No solutions or geometries presented in this note are essentially new; the purpose here is to look at them from a new viewpoint and re-interpret them, potentially as a basis on which to construct new solutions.

In the rest of the note, we will give more details of the picture explained above, and also discuss related matters. First, in section 2, we explain harmonic solutions, the Lunin-Mathur geometries, and the relation between them. Then, in section 3, we derive the harmonic functions that corresponds to the Lunin-Mathur geometry for the "helical" profile (1.4), and examine in detail the process of D4-charges getting transferred to the D6 center. We will also discuss various other matters such as how the constraint (1.5) is derived from a no-CTC (closed timelike curve) condition. In section 4, we confirm the harmonic functions derived in section 3 from the $6 \mathrm{~d}$ Lunin-Mathur geometry side. In section 5 , we discuss the dual CFT perspective of the whole process. We will also consider the spectral

\footnotetext{
${ }^{3}$ This delocalized charge is an example of "Cheshire charges" that can appear in the presence of monodromies [30].

${ }^{4}$ The delocalized charge here corresponds to the fundamental string charge carried by the KKM background in the context of [28].
} 
flow and fractional spectral flow of our solution, in both CFT and gravity. Finally, in section 6 , we discuss the implication of the result obtained in this note. Some details of the computation in the main text can be found in the appendix.

\section{Setup}

\subsection{Harmonic solutions}

Here we give a brief review of the harmonic solution, which represents multi-center blackhole/ring solutions in 4d/5d. Our purpose here is to introduce notation; for further detail, see [1-7] (for solutions with codimension-2 sources see also [22, 23]).

The most general supersymmetric solutions of ungauged $5 \mathrm{~d} \mathcal{N}=1$ supergravity with vector multiplets have been classified in [31] (see also [2, 4, 32]). When one applies this result to M-theory compactified on $T^{6}=T_{45}^{2} \times T_{67}^{2} \times T_{89}^{2}$ (the so-called STU model) and further assumes a tri-holomorphic U(1) symmetry [5], the general supersymmetric solution corresponds to the following 11d fields:

$$
\begin{aligned}
d s_{11}^{2} & =-Z^{-2 / 3}(d t+k)^{2}+Z^{1 / 3} d s_{\mathrm{GH}}^{2}+Z^{1 / 3}\left(Z_{1}^{-1} d x_{45}^{2}+Z_{2}^{-1} d x_{67}^{2}+Z_{3}^{-1} d x_{89}^{2}\right), \\
\mathcal{A}_{3} & =\left(B^{I}-Z_{I}^{-1}(d t+k)\right) \wedge J_{I}, \quad J_{1} \equiv d x^{4} \wedge d x^{5}, J_{2} \equiv d x^{6} \wedge d x^{7}, J_{3} \equiv d x^{8} \wedge d x^{9},
\end{aligned}
$$

where $I=1,2,3 ; Z \equiv Z_{1} Z_{2} Z_{3}$; and $d x_{45}^{2} \equiv\left(d x^{4}\right)^{2}+\left(d x^{5}\right)^{2}$ etc. Supersymmetry implies that all fields in (2.1) are written in terms of 3D harmonic functions

$$
\mathcal{H} \equiv\left(V, K^{I}, L_{I}, M\right)
$$

as follows. First, $d s_{\mathrm{GH}}^{2}$ is a 4-dimensional metric of a Gibbons-Hawking space given by

$$
d s_{\mathrm{GH}}^{2}=V^{-1}(d \psi+A)^{2}+V d \mathbf{y}^{2}, \quad \psi \cong \psi+4 \pi, \quad \mathbf{y}=\left(y_{1}, y_{2}, y_{3}\right) .
$$

The 1-form $A$ and the scalar $V$ depend on the coordinates $\mathbf{y}$ of the $\mathbb{R}^{3}$ base and satisfy

$$
d A=*_{3} d V
$$

where $*_{3}$ is the Hodge dual in flat $\mathbb{R}^{3}$. We generally denote 3 d vectors by boldface letters, such as $\mathbf{y}=\left(y_{i}\right), i=1,2,3$. The rest of the fields are:

$$
\begin{aligned}
B^{I} & =V^{-1} K^{I}(d \psi+A)+\xi^{I}, \quad d \xi^{I}=-*_{3} d K^{I}, \\
Z_{I} & =L_{I}+\frac{1}{2} C_{I J K} V^{-1} K^{J} K^{K}, \\
k & =\mu(d \psi+A)+\omega, \\
\mu & =M+\frac{1}{2} V^{-1} K^{I} L_{I}+\frac{1}{6} C_{I J K} V^{-2} K^{I} K^{J} K^{K},
\end{aligned}
$$

where $C_{I J K}=\left|\epsilon_{I J K}\right|$. All fields are assumed to depend only on $\mathbf{y}$ and not $\psi$ or $T^{6}$ coordinates. The physical fields in the solution, such as $Z_{I}, \mu$, are invariant under the 
"gauge transformation" [27]

$$
\begin{aligned}
V & \rightarrow V, \quad K^{I} \rightarrow K^{I}+c^{I} V, \\
L_{I} & \rightarrow L_{I}-C_{I J K} c^{J} K^{K}-\frac{1}{2} C_{I J K} c^{J} c^{K} V, \\
M & \rightarrow M-\frac{1}{2} c^{I} L_{I}+\frac{1}{12} C_{I J K}\left(c^{I} c^{J} c^{K} V+3 c^{I} c^{J} K^{K}\right)
\end{aligned}
$$

where $c^{I}$ are arbitrary constants. In the 5 d setup that includes $\psi$ as a coordinate, this is a gauge transformation for which the gauge transformation parameter depends on $\psi$ (it shifts the coefficient of $d \psi$ in $B^{I}$ ). However, in the $4 \mathrm{~d}$ context, such $\psi$ dependence is not allowed and the transformation (2.6) does change the charge [33].

As already stated, supersymmetry requires that $\mathcal{H}=\left(V, K^{I}, L_{I}, M\right)$ be harmonic functions in $\mathbb{R}^{3}$ :

$$
\triangle V=\triangle K^{I}=\triangle L_{I}=\triangle M=0, \quad \triangle \equiv \partial_{i} \partial_{i}
$$

The 1-form $\omega$ satisfies

$$
*_{3} d \omega=V d M-M d V+\frac{1}{2}\left(K^{I} d L_{I}-L_{I} d K^{I}\right) .
$$

Applying $d *_{3}$ on this equation we obtain the "integrability condition" [34] or the "bubble equation" [6]

$$
0=V \triangle M-M \triangle V+\frac{1}{2}\left(K^{I} \triangle L_{I}-L_{I} \triangle K^{I}\right) .
$$

Although the functions $V, K^{I}, L_{I}, M$ are harmonic, they have sources and the right-hand side of (2.9) has delta-function singularities. The integrability condition requires all such singularities cancel $[6] .^{5}$

Reducing the 11D solution (2.1) on the $\psi$ circle $S_{\psi}^{1}$, we obtain the following supersymmetric solution of type IIA supergravity:

$$
\begin{aligned}
d s_{10, \mathrm{str}}^{2} & =-\frac{1}{\sqrt{\mathcal{Q}}}(d t+\omega)^{2}+\sqrt{\mathcal{Q}} d \mathbf{x}^{2}+\frac{\sqrt{\mathcal{Q}}}{V}\left(Z_{1}^{-1} d x_{45}^{2}+Z_{2}^{-1} d x_{67}^{2}+Z_{3}^{-1} d x_{89}^{2}\right), \\
e^{2 \Phi} & =\frac{\mathcal{Q}^{3 / 2}}{V^{3} Z}, \quad B_{2}=\left(\frac{K^{I}}{V}-\frac{\mu}{Z_{I}}\right) J_{I},
\end{aligned}
$$

where $d s_{10 \text {,str }}^{2}$ is the string-frame metric and $\mathcal{Q} \equiv V\left(Z-\mu^{2} V\right)$. There are also RR potentials whose explicit form can be found e.g. in [22, appendix E] and [35]. The complexified Kähler modulus associated with $T_{89}^{2}$ is defined by

$$
\tau^{3}=\frac{R_{8} R_{9}}{\alpha^{\prime}}\left(B_{89}+i \sqrt{\operatorname{det} G_{a b}}\right)=\frac{R_{8} R_{9}}{\alpha^{\prime}}\left[\left(\frac{K^{3}}{V}-\frac{\mu}{Z_{3}}\right)+i \frac{\sqrt{\mathcal{Q}}}{Z_{1} V}\right]
$$

\footnotetext{
${ }^{5}$ For a discussion on the physical meaning of this condition for codimension-2 sources see [23].
} 
where $a, b=8,9$ and $R_{i}$ are the radii of the $x^{i}$ directions, $i=4, \ldots, 9$. Under the gauge transformation (2.6), this transforms as

$$
\tau^{3} \rightarrow \tau^{3}+\frac{R_{8} R_{9}}{\alpha^{\prime}} c^{3} .
$$

The moduli $\tau^{1}$ and $\tau^{2}$ for $T_{45}^{2}$ and $T_{67}^{2}$ are defined similarly, and the transformation under the gauge transformation (2.6) is similar.

Codimension-3 sources. The harmonic functions $\mathcal{H}=\left(V, K^{I}, L_{I}, M\right)$ can have sources that represent branes, which are D-branes in the IIA setup. If one assumes that all sources are of codimension 3 , the harmonic functions can be written as

$$
\mathcal{H}=h+\sum_{p=1}^{N} \frac{\Gamma_{p}}{\left|\mathbf{y}-\mathbf{a}_{p}\right|},
$$

where $\mathbf{a}_{p} \in \mathbb{R}^{3}(p=1, \ldots, N)$ specify the location of the codimension-3 sources where the harmonic functions become singular, and $h, \Gamma_{p}$ are constants.

The codimension-3 sources in the harmonic functions (2.13) represent branes in string/M-theory. For example, in the type IIA picture (2.10), the dictionary between the singularities in the harmonic functions and the D-brane sources is [3]

$$
\begin{aligned}
& K^{1} \leftrightarrow \mathrm{D} 4(6789) \quad L_{1} \leftrightarrow \mathrm{D} 2(45) \\
& V \leftrightarrow \mathrm{D} 6(456789), \quad K^{2} \leftrightarrow \mathrm{D} 4(4589), \quad L_{2} \leftrightarrow \mathrm{D} 2(67), \quad M \leftrightarrow \mathrm{D} 0 . \\
& K^{3} \leftrightarrow \mathrm{D} 4(4567) \quad L_{3} \leftrightarrow \mathrm{D} 2(89)
\end{aligned}
$$

The D-branes are partially wrapped on $T^{6}$ as indicated here and appear in $4 \mathrm{D}$ as pointlike (codimension-3) objects sourcing the harmonic functions. When lifted to M-theory, D4 becomes M5 wrapping $S_{\psi}^{1}$ and D2 becomes M2. D0 becomes momentum (P) along $S_{\psi}^{1}$ while D6 becomes Kaluza-Klein monopole (KKM) with $S_{\psi}^{1}$ being its special circle.

Depending on how to choose the base space (2.3), the harmonic solution can describe $4 \mathrm{~d}$ solutions or $5 \mathrm{~d}$ solutions. For example, take

$$
V=\frac{1}{r}, \quad K^{I}=0, \quad L_{I}=1+\frac{Q_{I}}{4 r}, \quad M=0, \quad r \equiv|\mathbf{y}| .
$$

In type IIA, this describes a 4 d black hole made of three stacks of D2-branes and a D6brane. Meanwhile, we can interpret this also as a $5 \mathrm{~d}$ black hole because, for this $V$, the base space (2.3) describes flat $\mathbb{R}^{4}$ via a Hopf fibration. Namely, the metric for flat $\mathbb{R}^{4}$ with coordinates $x_{m}, m=1,2,3,4$ can be written as

$$
\begin{aligned}
d s_{\mathbb{R}^{4}}^{2} & =d x_{m} d x_{m}=V^{-1}(d \psi+A)^{2}+V\left(d r^{2}+r^{2}\left(d \theta^{2}+\sin ^{2} \theta d \phi^{2}\right)\right), \\
V & =\frac{1}{r}, \quad A=(1+\cos \theta) d \phi,
\end{aligned}
$$

where

$$
x_{1}+i x_{2}=2 \sqrt{r} \sin \frac{\theta}{2} e^{i \frac{\psi}{2}}, \quad x_{3}+i x_{4}=2 \sqrt{r} \cos \frac{\theta}{2} e^{i\left(\frac{\psi}{2}+\phi\right)},
$$


with $\psi \cong \psi+4 \pi, \phi \cong \phi+2 \pi$. The Cartesian coordinate $\mathbf{y}$ in the $3 \mathrm{~d}$ base is

$$
y_{1}+i y_{2}=r \sin \theta e^{i \phi}, \quad y_{3}=r \cos \theta .
$$

Lifted to M-theory along the $\psi$ direction, this harmonic solution becomes a $5 \mathrm{~d}$ black hole made of three stacks of M2-branes, the D6-brane becoming the origin of the $\mathbb{R}^{4}$. This $5 \mathrm{~d}$ black hole can be dualized (using the duality of appendix A) into the original StromingerVafa black hole [36] in a type IIB frame, where the M2/D2 charges are mapped into D1, D5, and $\mathrm{P}$ charges.

Codimension-2 sources. Harmonic functions can have other kinds of source. They can have a singularity along a curve in $\mathbb{R}^{3}$ and have non-trivial monodromy around it. We refer to such singularities as codimension-2 sources. Note that this is genuinely different from the codimension-3 source discussed above; we can have a continuous distribution of codimension- 3 sources along a curve, but we will still refer to them as codimension-3 sources.

One situation for codimension-2 sources to appear is when branes undergo a supertube transition [21], gaining dimension (or losing codimension). For example, the following supertube transition is possible:

$$
\mathrm{D} 2(45)+\mathrm{D} 2(67) \stackrel{\text { puff out }}{\longrightarrow} \mathrm{NS} 5(4567 \lambda)+\mathrm{P}(\lambda),
$$

where in the final configuration there is an NS5-brane along internal (4567) directions and a closed curve $\mathcal{C}$ in $\mathbb{R}^{3}$ parametrized by $\lambda$, and momentum along $\lambda$. By looking at the expression for $B_{2}$ in (2.10), we see that, if $V^{-1} K^{3}$ has a monodromy around $\mathcal{C}$, there will be an NS5-brane along $\mathcal{C}$ (and 4567). (More precisely it is $V^{-1} K^{3}-Z_{3}^{-1} \mu$ that must be monodromic, but in the current note we assume that $Z_{I}, \mu$ appearing in the metric are single-valued and therefore it is the monodromy of $V^{-1} K^{3}$ that is relevant.) As it turns out, in this case $M$ also becomes monodromic. In general, given codimension-2 brane sources, which harmonic functions to become monodromic is a non-trivial matter that depends on the physical situation in question.

\subsection{Lunin-Mathur geometries}

The Lunin-Mathur geometry [24, 25] is a solution of type IIB supergravity in $\mathbb{R}_{t} \times \mathbb{R}_{1234}^{4} \times$ $S_{y}^{1} \times T_{6789}^{4}$ and represents microstates $[37,38]$ of the D1-D5 2-charge system. The solution is parametrized by profile functions $g_{m}(\lambda), m=1,2,3,4$ satisfying $g_{m}(\lambda+L)=g_{m}(\lambda)$, which describe the shape inside $\mathbb{R}^{4}$ of the KKM dipole produced by the supertube transition of D1(y) and D5(y6789) branes. ${ }^{6}$ Just as (2.19), we can describe this by the following diagram:

$$
\mathrm{D} 1(y)+\mathrm{D} 5(y 6789) \stackrel{\text { puff out }}{\longrightarrow} \mathrm{KKM}(6789 \lambda, y)+\mathrm{P}(\lambda),
$$

where $\operatorname{KKM}(6789 \lambda, y)$ denotes the KKM dipole with $y$ being the special circle.

\footnotetext{
${ }^{6}$ We do not consider $g_{A}$ with $A \geq 5$ [39], which describe other possible dipole charges produced by the supertube transition.
} 
The explicit form of the 10d string-frame metric of the Lunin-Mathur geometry is

$$
d s_{10}^{2}=-\frac{2}{\sqrt{Z_{1} Z_{2}}}(d v+\boldsymbol{\beta})\left(d u+\boldsymbol{\omega}+\frac{\mathcal{F}}{2}(d v+\boldsymbol{\beta})\right)+\sqrt{Z_{1} Z_{2}} d s_{\mathbb{R}^{4}}^{2}+\sqrt{\frac{Z_{1}}{Z_{2}}} d s_{T^{4}}^{2},
$$

where

$$
\begin{array}{rlrl}
Z_{1} & =\frac{Q_{5}}{L} \int_{0}^{L} d \lambda \frac{\left|\partial_{\lambda} \vec{g}(\lambda)\right|^{2}}{|\vec{x}-\vec{g}(\lambda)|^{2}}, & Z_{2} & =\frac{Q_{5}}{L} \int_{0}^{L} \frac{d \lambda}{|\vec{x}-\vec{g}(\lambda)|^{2}}, \\
\mathbf{A} & =-\frac{Q_{5}}{L} d x^{j} \int_{0}^{L} d \lambda \frac{\partial_{\lambda} g_{j}(\lambda)}{|\vec{x}-\vec{g}(\lambda)|^{2}}, & d \mathbf{B}=-*_{4} d \mathbf{A}, \\
Q_{1} & =\frac{Q_{5}}{L} \int_{0}^{L} d \lambda\left|\partial_{\lambda} \vec{g}(\lambda)\right|^{2}, & & \\
\boldsymbol{\beta} & =\frac{-\mathbf{A}+\mathbf{B}}{\sqrt{2}}, & \boldsymbol{\omega} & =\frac{-\mathbf{A}-\mathbf{B}}{\sqrt{2}}
\end{array}
$$

and $d s_{\mathbb{R}^{4}}^{2}=d x_{m} d x_{m}, m=1,2,3,4$ is the flat $\mathbb{R}^{4}$ metric with coordinates $\vec{x}=\left(x_{m}\right)$. For later convenience, we have written the metric (2.21a) in the general form given in [40, appendix E] which represents the 3-charge solution. In the 2-charge case we are considering, we must set $\mathcal{F}=0$. Also, we have taken the decoupling limit and dropped " 1 " from $Z_{1,2}$. The coordinates $u, v$ are related to time $t$ and the coordinate $y$ of the compact circle $S_{y}^{1}$ of radius $R_{y}$ as

$$
u=\frac{1}{\sqrt{2}}(t-y), \quad v=\frac{1}{\sqrt{2}}(t+y) .
$$

The periodicity $L$ is related to $R_{y}$ as $L=2 \pi Q_{5} / R_{y}$. The quantities $Q_{1}, Q_{5}$ are related to the quantized D1 and D5 numbers $N_{1}, N_{5}$ by

$$
Q_{1}=\frac{N_{1} g_{s} \alpha^{\prime 3}}{v_{4}}, \quad Q_{5}=N_{5} g_{s} \alpha^{\prime}
$$

where $(2 \pi)^{4} v_{4}$ is the coordinate volume of $T^{4}$. Finally, $*_{4}$ is the Hodge dual in flat $\mathbb{R}^{4}$ with coordinates $x_{m}$.

The simplest example of the profile, which has already been introduced in the introduction, is a circle in the $x_{1}-x_{2}$ plane:

$$
g_{1}+i g_{2}=a e^{i k \Omega \lambda}, \quad g_{3}+i g_{4}=0,
$$

where $a>0, k \in \mathbb{Z}_{>0}$ and

$$
\Omega \equiv \frac{2 \pi}{L}=\frac{R_{y}}{Q_{5}} .
$$

Substituting this into (2.22), we find

$$
\begin{aligned}
Z_{1} & =\frac{Q_{1}}{4 \Sigma}, \quad Z_{2}=\frac{Q_{5}}{4 \Sigma}, \\
Q_{1} & =Q_{5} a^{2} k^{2} \Omega^{2}, \\
\mathbf{A} & =-\frac{1}{2} Q_{5} k \Omega\left(\frac{s^{2}+a^{2}+w^{2}}{4 \Sigma}-1\right) d \widetilde{\phi}, \\
\mathbf{B} & =+\frac{1}{2} Q_{5} k \Omega\left(\frac{s^{2}-a^{2}+w^{2}}{4 \Sigma}-1\right) d \widetilde{\psi},
\end{aligned}
$$


where

$$
\Sigma \equiv \frac{1}{4} \sqrt{\left[(s+a)^{2}+w^{2}\right]\left[(s-a)^{2}+w^{2}\right]}
$$

and we defined the polar coordinates $(s, \widetilde{\phi}),(w, \widetilde{\psi})$ via

$$
x_{1}+i x_{2}=s e^{i \widetilde{\phi}}, \quad x_{3}+i x_{4}=w e^{i \widetilde{\psi}}
$$

with $s, w \geq 0$ and $\widetilde{\phi} \cong \widetilde{\phi}+2 \pi, \widetilde{\psi} \cong \widetilde{\psi}+2 \pi$.

\subsection{Relation to harmonic solutions}

By appropriate duality transformations (see appendix A), we can map the harmonic solutions in the M/type-IIA frame in section 2.1 into the type IIB D1-D5 frame in section 2.2. Here let us study the relation between the fields in the Lunin-Mathur geometries and the harmonic functions in the harmonic solutions.

As discussed in [41], the harmonic solutions in the D1-D5 frame is

$$
\begin{aligned}
d s_{\mathrm{IIB}}^{2} & =-\frac{1}{Z_{3} \sqrt{Z_{1} Z_{2}}}(d t+\kappa)^{2}+\frac{Z_{3}}{\sqrt{Z_{1} Z_{2}}}\left(d z+A^{3}\right)^{2}+\sqrt{Z_{1} Z_{2}} d s_{\mathrm{GH}}^{2}+\sqrt{\frac{Z_{1}}{Z_{2}}} d s_{T^{4}}^{2}, \\
\kappa & =\mu(d \psi+A)+\omega, \quad A^{3}=B^{3}-\frac{1}{Z_{3}}(d t+\kappa),
\end{aligned}
$$

where $B^{3}$ is the one given in (2.5a). On the other hand, the first part (the first term) of the Lunin-Mathur solution (2.21a) can be rewritten as

$$
d s_{10}^{2}=-\frac{(d t-\mathbf{A})^{2}}{Z_{3} \sqrt{Z_{1} Z_{2}}}+\frac{Z_{3}}{\sqrt{Z_{1} Z_{2}}}\left(d y+d t+\mathbf{B}-\mathbf{A}-\frac{d t-\mathbf{A}}{Z_{3}}\right)^{2}+\cdots
$$

where $Z_{3} \equiv 1-\mathcal{F} / 2$ (actually $Z_{3}=1$ in the D1-D5 case). By comparing (2.30) and (2.32), we read off the relation between the 1-forms $\mathbf{A}, \mathbf{B}$ of the Lunin-Mathur geometry and the harmonic functions:

$$
\begin{aligned}
-\mathbf{A} & \leftrightarrow \kappa=\mu(d \psi+A)+\omega, \\
-\mathbf{A}+\mathbf{B} & \leftrightarrow B^{3}=V^{-1} K^{3}(d \psi+A)+\xi^{3}
\end{aligned}
$$

and $y+t \leftrightarrow z$. With this dictionary, we can read off harmonic functions that correspond to a Lunin-Mathur solution, and vice versa.

Comparison of (2.21a) and (2.30) also means that the flat $\mathbb{R}^{4}$ in which the D1-D5 profile $g_{m}(\lambda)$ lives should be written as the Hopf fibration (2.16). ${ }^{7}$ Generically, the profile in $\mathbb{R}^{4}$ is going along some curve $\mathcal{C}$ in the $\mathbb{R}^{3}$ base, at the same time moving in the $\psi$ fiber. Because the harmonic solutions are independent of the $\psi$ fiber, we must smear the profile along $\psi$. The exception is when the profile is purely in the $\psi$ direction and $\mathcal{C}$ is a point in the base. One such exceptional example is the circular profile $(2.25)$, for which $\mathcal{C}$ corresponds to the point on the $y_{3}$ axis, $r=a^{2} / 4, \theta=\pi\left(y_{3}=-a^{2} / 4\right)$.

\footnotetext{
${ }^{7}$ Another possibility is to compactify $\mathbb{R}^{4}$ trivially to $\mathbb{R}^{3} \times S_{\psi}^{1}$ by taking $V=1, A=0$. Codimension- 2 harmonic solutions obtained this way was discussed in [22].
} 
We can see which harmonic functions get sources as follows. The $\lambda$ direction of the profile in (2.20) can now be along $\psi$ or $\mathcal{C} \subset \mathbb{R}^{3}$, so we have two kinds of dipole charge that can be produced by the supertube transition:

$$
\mathrm{D} 1(y)+\mathrm{D} 5(y 6789) \stackrel{\text { puff out }}{\longrightarrow}\left\{\begin{array}{l}
\operatorname{KKM}(6789 \psi, y)+\mathrm{P}(\psi) \\
\operatorname{KKM}(6789 \mathcal{C}, y)+\mathrm{P}(\mathcal{C})
\end{array}\right.
$$

After the duality transformation of appendix A, this is mapped into the following process in IIA:

$$
\mathrm{D} 2(45)+\mathrm{D} 2(67) \stackrel{\text { puff out }}{\longrightarrow}\left\{\begin{array}{l}
\mathrm{D} 4(4567)+\mathrm{D} 0 \\
\mathrm{NS} 5(4567 \mathcal{C})+\mathrm{P}(\mathcal{C})
\end{array}\right.
$$

The first line means that $K^{3}$ will have codimension-3 sources for D4(4567). The codimension-3 charge is continuously distributed along $\mathcal{C}$, and its local density is proportional to the "speed" of the profile going along $\psi$. In addition, $K^{3}$ will have codimension- 2 sources for NS5(4567C); namely, $V^{-1} K^{3}$ will have a constant additive monodromy as we mover around $\mathcal{C}$ and the constant is proportional to the NS5-brane charge. Also, $M$ will have codimension-3 sources for D0, while momentum $\mathrm{P}(\mathcal{C})$ will be encoded in the 1-form $\omega$.

We can confirm that the non-trivial data of the Lunin-Mathur geometry are encoded in $K^{3}$ and $M$ from the relation (2.33). The duality equation between $A$ and $B$ (the second equation in $(2.22 \mathrm{~b}))$ means that $d(-\mathbf{A}+\mathbf{B})=d B^{3}$ is the self-dual part of $d(-\mathbf{A})=d k$, namely $\left(1+*_{4}\right) d k=d B^{3}$. From this, we can derive

$$
\begin{aligned}
\mu *_{3} d A+d \omega-V *_{3} d \mu & =V^{-1} K^{3} d A+d \xi^{3}, \\
-d \mu+\mu V^{-1} d V+V^{-1} *_{3} d \omega & =-d\left(V^{-1} K^{3}\right) .
\end{aligned}
$$

Here we used the relations between $*_{4}$ and $*_{3}$ on the Gibbons-Hawking space (2.3),

$$
\begin{aligned}
*_{4} \lambda_{(2)} & =V^{-1}(d \psi+A) \wedge *_{3} \lambda_{(2)}, & *_{4}\left((d \psi+A) \wedge \theta_{(1)}\right) & =V *_{3} \theta_{(1)}, \\
*_{4} \theta_{(1)} & =(d \psi+A) \wedge *_{3} \theta_{(1)}, & *_{4}(d \psi+A) & =V^{2} d^{3} y,
\end{aligned}
$$

where $\lambda_{(2)}$ is a 2 -form and $\theta_{(1)}$ is a 1 -form in $\mathbb{R}^{3}$. The two equations in (2.36) are $*_{3}$ of each other. By acting with $d$ on (2.36a) we can derive

$$
d *_{3} d M=0, \quad \mu=M+\frac{1}{2} V^{-1} K^{3} .
$$

Plugging (2.38) into (2.36b), we find

$$
*_{3} d \omega=V d M-M d V-\frac{1}{2} d K^{3} .
$$

Equations (2.38) and (2.39) are consistent with the relations (2.5d) and (2.8) under the identification

$$
V=\frac{1}{r}, \quad L_{1}=Z_{1}, \quad L_{2}=Z_{2}, \quad L_{3}=1, \quad K^{1}=K^{2}=0 .
$$

So, the non-trivial data of the solution will be in $K^{3}$ and $M$.

Using the above dictionary (2.33), we can readily check that the Lunin-Mathur solution based on the circular profile (2.25) corresponds to a harmonic solution with the harmonic functions given in (1.2) in the introduction. 


\section{Codimension-2 Lunin-Mathur solution}

Here we explicitly construct harmonic functions that describe a Lunin-Mathur geometry with both dipole charges in (2.34) (or equivalently (2.35)). Specifically, we consider the following profile:

$$
g_{1}+i g_{2}=a e^{i k \Omega \lambda}, \quad g_{3}+i g_{4}=b e^{-i k^{\prime} \Omega \lambda}
$$

with $a, b \geq 0,{ }^{8}$ and $k, k^{\prime} \in \mathbb{Z}_{>0} \cdot{ }^{9}$ We have already discussed this profile in (1.4) in the introduction. When smeared along and reduced on $\psi$, this gives a circular ring in the base $\mathbb{R}^{3}$ :

$$
\rho \equiv \sqrt{y_{1}^{2}+y_{2}^{2}}=R, \quad y_{3}=c,
$$

where

$$
R=\frac{a b}{2}, \quad c=\frac{-a^{2}+b^{2}}{4} .
$$

This is the codimension- 2 solution that we would like to construct and study.

Of course, we can derive the harmonic functions "top-down" by starting with the Lunin-Mathur geometry in $6 \mathrm{~d}$ with the profile (3.1), smearing and reducing it to $4 \mathrm{~d} / 5 \mathrm{~d}$, and then reading off the harmonic function. However, here we go "bottom-up" by directly constructing the harmonic functions based on the expected charges that they must represent. In the next section, we will confirm the result from the top-down viewpoint starting from the Lunin-Mathur geometry.

The profile (3.1) is going in the angular directions $\phi, \psi$ as

$$
\phi=-\left(k+k^{\prime}\right) \Omega \lambda, \quad \psi=2 k \Omega \lambda .
$$

Therefore, by the argument below (2.34) we will have $-\left(k+k^{\prime}\right)$ units of NS5 charges and $k$ units of D4(4567) charges, encoded in harmonic functions.

As mentioned in the introduction, $a$ and $b$ are constrained to satisfy (1.5) (this will be shown in (3.47)). If we fix the charges of the system, $Q_{1}$ and $Q_{5}$, and also the parameters $k$ and $k^{\prime}$, then the ring will be on a spheroid in $\mathbb{R}^{3}$ for any values of $a$ and $b$. More precisely, the position of the ring $\rho=R=a b / 2, y_{3}=c=\left(-a^{2}+b^{2}\right) / 4$ can be shown to satisfy

$$
\left(\frac{y_{3}-y_{3}^{(0)}}{A}\right)^{2}+\left(\frac{\rho}{B}\right)^{2}=1
$$

where

$$
A \equiv \frac{\mathcal{N}}{8}\left(\frac{1}{k^{2}}+\frac{1}{k^{\prime 2}}\right), \quad B \equiv \frac{\mathcal{N}}{4 k k^{\prime}}, \quad y_{3}^{(0)} \equiv \frac{\mathcal{N}}{8}\left(\frac{1}{k^{\prime 2}}-\frac{1}{k^{2}}\right), \quad \mathcal{N} \equiv \frac{Q_{1}}{Q_{5} \Omega^{2}}=\frac{Q_{1} Q_{5}}{R_{y}^{2}}
$$

\footnotetext{
${ }^{8}$ We could make $a, b$ complex but that does not make a difference after smearing.

${ }^{9}$ The negative sign in front of $k^{\prime}$ in $g_{3}+i g_{4}$ is because of the holographic dictionary [42]; see section 5 .
} 
The surface (3.5) is a spheroid, whose symmetry axis is the $y_{3}$ axis. The origin $\mathbf{y}=0$ is a focal point of the ellipse on the cross section that contains the $y_{3}$ axis. Because $A \geq B$, the spheroid is prolate.

We will be interested in the process of starting with $a>0, b=0$ and then increasing $b$, finally ending with $a=0, b>0$. When $a>b$, the $y_{3}$ coordinate of the ring is $c<0$. We call this the "southern" case, because the ring is like a latitude line on the southern hemisphere of the spheroid. When $a<b$, the $y_{3}$ coordinate of the ring is $c>0$. We call this the "northern" case. However, the word "southern"/"northern" should not be taken literally, because the center of the spheroid is not at $y_{3}=0$. See figure 1 .

As the extreme cases, if $a>0, b=0$ (the "south pole" limit), the $4 \mathrm{~d}$ profile is a circle of radius $a$ on the $x_{1}-x_{2}$ plane discussed in $(2.25)$, which projects in $\mathbb{R}^{3}$ onto a point on the negative $y_{3}$ axis $\left(y_{3}=-a^{2} / 4\right)$. In this case, the $\phi$ circle shrinks and there is no NS5 charge. So, this limit gives a codimension- 3 solution. If $a=0, b>0$ (the "north pole" limit), on the other hand, the profile is a circle of radius $b$ on the $x_{3}-x_{4}$ plane, which projects onto a point on the positive $y_{3}$ axis $\left(y_{3}=b^{2} / 4\right)$. So, this limit also gives a codimension- 3 solution. In this case, the shrinking cycle is not $\phi$ or $\psi$ but their linear combination because of the nontrivial Hopf fibration.

\subsection{Building blocks}

To find the harmonic solution that corresponds to the profile (3.1), we start by constructing appropriately normalized harmonic functions that have codimension- 2 and codimension- 3 sources along the ring (3.2), as building blocks.

It is useful to introduce toroidal coordinates $(u, \sigma, \phi)$, adapted for a ring sitting at $\sqrt{y_{1}^{2}+y_{2}^{2}}=R, y_{3}=c$ :

$$
y_{1}=\frac{\sqrt{u^{2}-1}}{u-\cos \sigma} R \cos \phi, \quad y_{2}=\frac{\sqrt{u^{2}-1}}{u-\cos \sigma} R \sin \phi, \quad y_{3}^{\prime} \equiv y_{3}-c=\frac{\sin \sigma}{u-\cos \sigma} R,
$$

where $1 \leq u<\infty, \phi \cong \phi+2 \pi, \sigma \cong \sigma+2 \pi$. The ring sits at $u=\infty$, while $u=1, \sigma=0$ corresponds to $r=|\mathbf{y}| \rightarrow \infty$. $\phi$ is the angle along the ring while $\sigma$ is the angle going around the ring. See figure 2 for a graphical description. The flat $3 \mathrm{~d}$ metric can be written as

$$
d \mathbf{y}^{2}=d y_{1}^{2}+d y_{2}^{2}+d y_{3}^{\prime 2}=\frac{R^{2}}{(u-\cos \sigma)^{2}}\left[\frac{d u^{2}}{u^{2}-1}+d \sigma^{2}+\left(u^{2}-1\right) d \phi^{2}\right] .
$$

The inverse relations are

$$
\cos \sigma=\frac{\mathbf{y}^{\prime 2}-R^{2}}{\Lambda}, \quad u=\frac{\mathbf{y}^{\prime 2}+R^{2}}{\Lambda}, \quad \Lambda^{2}=\left(\mathbf{y}^{\prime 2}-R^{2}\right)^{2}+4 R^{2} y_{3}^{\prime 2},
$$

where $\mathbf{y}^{\prime 2}=y_{1}^{2}+y_{2}^{2}+y_{3}^{\prime 2}$.

Near the ring, $u \rightarrow \infty$,

$$
\sqrt{y_{1}^{2}+y_{2}^{2}} \approx R+\varrho \cos \sigma, \quad y_{3}^{\prime}=y_{3}-c \approx \varrho \sin \sigma, \quad \varrho \equiv \frac{R}{u} .
$$

Namely, $(\varrho, \sigma)$ are plane polar coordinates near the position of the ring. 

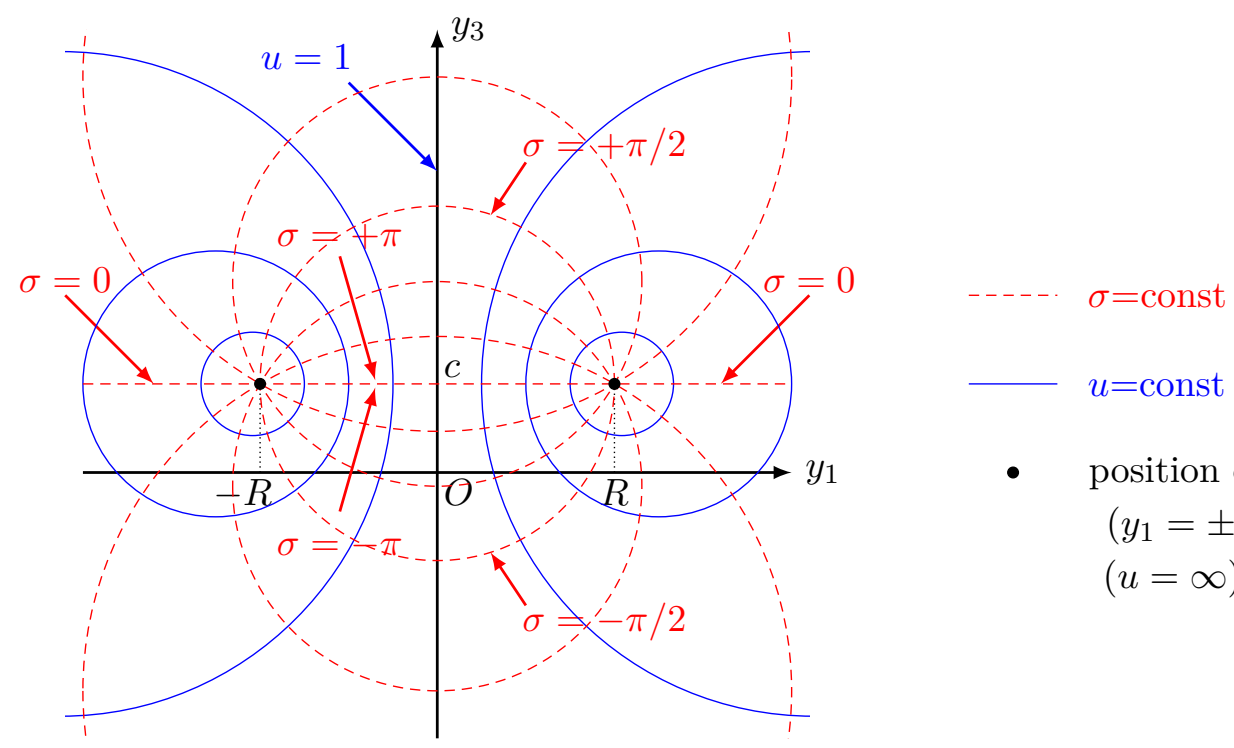

- $\quad$ position of the ring

$$
\begin{aligned}
& \left(y_{1}= \pm R, y_{3}=c\right) \\
& (u=\infty)
\end{aligned}
$$

Figure 2. Toroidal coordinates (on the $y_{2}=0$ plane).

Let us first introduce $H$, which is a single-valued harmonic function with codimension-3 sources uniformly distributed along the ring. Explicitly, it is given by

$$
H=\frac{1}{R} \sqrt{\frac{u-\cos \sigma}{2}} P_{-1 / 2}(u)=\frac{4 \mathcal{I}_{00}(P, Q),}{s^{2}+a^{2}+w^{2}+b^{2}}
$$

where $P_{n}(u)$ is the Legendre function and we defined the following quantities:

$$
\begin{aligned}
P & \equiv \frac{2 s a}{s^{2}+a^{2}+w^{2}+b^{2}}, \quad Q \equiv \frac{2 w b}{s^{2}+a^{2}+w^{2}+b^{2}}, \\
\mathcal{I}_{m n}(P, Q) & \equiv \int_{0}^{2 \pi} \frac{d \mu}{2 \pi} \int_{0}^{2 \pi} \frac{d \nu}{2 \pi} \frac{(P \cos \mu)^{m}(Q \cos \nu)^{n}}{1-P \cos \mu-Q \cos \nu} .
\end{aligned}
$$

For other expressions of $H$, see appendix $\mathrm{C}$. The behavior of $H$ near the ring and near infinity is

$$
H= \begin{cases}\frac{\log (8 u)}{\pi R}+\mathcal{O}\left(\frac{1}{u}\right) & \text { (near the ring, } u \rightarrow \infty), \\ \frac{1}{r}+\mathcal{O}\left(\frac{1}{r^{2}}\right) & \text { (near infinity, } r \rightarrow \infty) .\end{cases}
$$

Because $H$ is harmonic, $\triangle H$ vanishes except on the ring. The first line of (3.13) implies that $\triangle H$ has delta-function singularities uniformly smeared along the ring, and the total charge, as measured by the coefficient of $1 / r$ for large $r$, is unity.

Next, we want a harmonic function - call it $\gamma$ - which has the following monodromy around the ring:

$$
\gamma(u, \sigma+2 \pi) \rightarrow \gamma(u, \sigma)+1
$$


Actually, let us be more general for a bit and consider a harmonic function $\gamma$ with the monodromy $\gamma \rightarrow \gamma+1$ around a general closed curve $\mathcal{C}$ in $\mathbb{R}^{3}$. For that, it is convenient to introduce a 1 -form

$$
\alpha=d y_{i} \int_{0}^{2 \pi} \frac{d \mu}{4 \pi} \frac{\partial_{\mu} f_{i}(\mu)}{|\mathbf{y}-\mathbf{f}(\mu)|}
$$

where $f_{i}(\mu), 0 \leq \mu \leq 2 \pi$, is a $3 \mathrm{~d}$ profile function that parametrize the curve $\mathcal{C}$ (this $\alpha$ is a $3 \mathrm{~d}$ version of $\mathbf{A}$ defined in (2.22b)). The 1 -form $\alpha$ is independent of how we parametrize $\mathbf{f}(\mu)$. It is not difficult to show that, if we define $\gamma$ by the condition

$$
d \gamma=*_{3} d \alpha,
$$

then $\gamma$ is harmonic and has the desired monodromy $\gamma \rightarrow \gamma+1$ as we go around $\mathcal{C}$. One can also show [22] that $\triangle \gamma$, which could have delta-function singularities on $\mathcal{C}$, is identically zero:

$$
\triangle \gamma=0 \quad \text { (no delta function) }
$$

This will be important when we impose the integrability condition.

In the present case, curve $\mathcal{C}$ is the circular ring (3.2). Being careful to the orientation of the angle variable $\sigma$ relative to the ring (see figure 2), we take the $3 \mathrm{~d}$ profile function to be

$$
f_{1}+i f_{2}=R e^{-i \mu}, \quad f_{3}=c .
$$

The 1-form $\alpha$ for this profile is found to be [43]

$$
\alpha(u, \sigma)=\frac{a(u)}{\sqrt{u-\cos \sigma}} d \phi, \quad a(u)=-\frac{R}{8 \sqrt{2}} \frac{u^{2}-1}{u^{3 / 2}}{ }_{2} F_{1}\left(\frac{3}{4}, \frac{5}{4} ; 2 ; 1-u^{-2}\right) .
$$

The function $\gamma$ that satisfies (3.16) is

$$
\gamma(u, \sigma)=\frac{1}{R}\left[\frac{a(u)}{\sqrt{u-1}} \mathbf{F}\left(\frac{\sigma}{2} \mid-\frac{2}{u-1}\right)-2 \sqrt{u-1} a^{\prime}(u) \mathbf{E}\left(\frac{\sigma}{2} \mid-\frac{2}{u-1}\right)\right],
$$

where $\mathbf{F}(\phi \mid m)$ and $\mathbf{E}(\phi \mid m)$ are the elliptic integrals of the first and second kinds, respectively. ${ }^{10}$ One can check the monodromy (3.14) from the periodicity of the elliptic integrals. This $\gamma$ vanishes at infinity $r=|\mathbf{y}| \rightarrow \infty$, which corresponds to $\sigma=0, u \rightarrow 1$ (and if we go to other branches $\sigma=2 \pi n, u \rightarrow 1$ with $n \in \mathbb{Z}$ then $\gamma \rightarrow n$; a related formula is (C.21)). The near-ring behavior of $\gamma$ is found to be

$$
\gamma(u, \sigma)=\frac{\sigma}{2 \pi}+\mathcal{O}\left(\frac{1}{u}\right)
$$

\footnotetext{
${ }^{10}$ We follow the Mathematica convention for the arguments of elliptic integrals.
} 


\subsection{Codimension-2 source}

Using $H, \gamma$ defined above, let us find harmonic functions that represent the Lunin-Mathur geometry with the profile (3.1). First, we have

$$
V=\frac{1}{r}, \quad Z_{1}=L_{1}=\frac{Q_{1}}{4} H, \quad Z_{2}=L_{2}=\frac{Q_{5}}{4} H, \quad L_{3}=1, \quad K^{1}=K^{2}=0 .
$$

$V$ is always the same for the Hopf fibration, while $Z_{1,2}$ are determined so that the total charge is the same as in the south pole limit (1.2) (or (2.27a)) but now the charges must distributed uniformly along the ring. The other harmonic functions are fixed by (2.40).

Before studying what singularities $K^{3}$ must have, let us discuss its normalization. When there are $n$ units of $\operatorname{KKM}(6789 \psi, y)$ charge at $r=0, K^{3}$ has the following codimension-3 center:

$$
K^{3} \supset \frac{R_{y} n}{2 r}=\frac{Q_{5} \Omega n}{2 r},
$$

where we used (2.26). On the other hand, when there are $n^{\prime}$ units of $\operatorname{KKM}(6789 \mathcal{C}, y)$ charge lying along curve $\mathcal{C}$, then $V^{-1} K^{3}$ has the following monodromy as we go around $\mathcal{C}$ :

$$
\left.\Delta\left(V^{-1} K^{3}\right) \equiv V^{-1} K^{3}\right|_{\sigma+2 \pi}-\left.V^{-1} K^{3}\right|_{\sigma}=\frac{R_{y} n^{\prime}}{R_{\psi}}=\frac{Q_{5} \Omega n^{\prime}}{2}
$$

where $R_{\psi}=2$. These normalizations can be derived by standard arguments (see e.g. [44]).

From (3.4), we know that we have $-\left(k+k^{\prime}\right)$ units of $\operatorname{KKM}(6789 \mathcal{C}, y)$ lying along $\phi$. So, $K^{3}$ must have the monodromy (3.24) with $n^{\prime}=-\left(k+k^{\prime}\right)$. Clearly, this requirement is satisfied if we take $K^{3}$ to be:

$$
K^{3}=-\frac{Q_{5} \Omega\left(k+k^{\prime}\right)}{2}\left(\gamma V+\widetilde{K}^{3}\right),
$$

where $\widetilde{K^{3}}$ is a single-valued function. For $K^{3}$ to be harmonic, $\widetilde{K^{3}}$ must satisfy

$$
\triangle \widetilde{K}^{3}=-\triangle(\gamma V)=-\partial_{i} \gamma \partial_{i} V=-\epsilon_{i j k} \partial_{j} \alpha_{k} \partial_{i} V,
$$

where in the second equality we used that $\gamma, V$ are harmonic and in the last equality we used (3.16). A special solution of this Poisson equation is

$$
\widetilde{K}^{3}=-\int_{0}^{2 \pi} \frac{d \mu}{4 \pi} \frac{\epsilon_{i j k} y_{i} f_{j} \partial_{\mu} f_{k}}{r|\mathbf{f}||\mathbf{y}-\mathbf{f}|(r+|\mathbf{f}|+|\mathbf{y}-\mathbf{f}|)},
$$

where $\alpha$ and $\mathbf{f}$ are given in (3.15) and (3.18). In the present case where $f_{i}$ is given by (3.18), this can be evaluated as

$$
\begin{aligned}
\widetilde{K}^{3} & =-\frac{R}{r|\mathbf{f}|} \int_{0}^{2 \pi} \frac{d \mu}{4 \pi} \frac{c \rho \cos (\mu+\phi)-R y_{3}}{|\mathbf{y}-\mathbf{f}|(|\mathbf{f}|+|\mathbf{y}-\mathbf{f}|+r)} \\
& =-\frac{4 a b V}{\left(a^{2}+b^{2}\right)\left(s^{2}+w^{2}+a^{2}+b^{2}\right)^{2}} \int_{0}^{2 \pi} \frac{d \mu^{\prime}}{4 \pi} \frac{\left(b^{2}-a^{2}\right) s w \cos \mu^{\prime}+a b\left(s^{2}-w^{2}\right)}{\sqrt{X}(1+\sqrt{X})}
\end{aligned}
$$

where $\mu^{\prime}=\mu+\phi, X \equiv 1-\left(P^{2}+Q^{2}\right)-2 P Q \cos \mu^{\prime}(P, Q$ were defined in (3.12a)). In the first equality we went to cylindrical coordinates $\left(\rho, \phi, y_{3}\right)$ in $\mathbb{R}^{3}$ and in the second equality 
we used relations such as (B.8e), (B.9), and (B.10a). One can show (see appendix C.4) that this can be written in terms of $\mathcal{I}_{m n}(P, Q)$ as

$$
\begin{aligned}
\widetilde{K}^{3} & =-\frac{b^{2} \mathcal{I}_{10}(P, Q)-a^{2} \mathcal{I}_{01}(P, Q)}{a^{2}+b^{2}} V \\
& =-\frac{1}{a^{2}+b^{2}} V \int_{0}^{2 \pi} \frac{d \mu}{2 \pi} \int_{0}^{2 \pi} \frac{d \nu}{2 \pi} \frac{b^{2} P \cos \mu-a^{2} Q \cos \nu}{1-P \cos \mu-Q \cos \nu}
\end{aligned}
$$

So, we have fixed the form of $K^{3}$ with the desired codimension- 2 source to be

$$
K^{3}=-\frac{Q_{5} \Omega}{2}\left(k+k^{\prime}\right)\left(\gamma-\frac{b^{2} \mathcal{I}_{10}-a^{2} \mathcal{I}_{01}}{a^{2}+b^{2}}\right) V+K_{(3)}^{3}
$$

where $K_{(3)}^{3}$ is a single-valued harmonic function containing only codimension-3 sources, which we turn to next. $\mathcal{I}_{10}, \mathcal{I}_{01}$ can be expressed in terms of elliptic integrals; see appendix C.

\subsection{Codimension-3 sources}

We want $K^{3}$ to also have $k$ units of codimension-3 D4-brane charge along the ring, as discussed in (3.4). We cannot simply set $K_{(3)}^{3}$ to $k H$, because the first term of (3.30) also already contains some codimension-3 charge, which we must take into account.

Codimension-3 sources in a harmonic function will appear as $\delta$-function singularities when we act with $\triangle$ on it. So, let us examine the expression

$$
\triangle\left[\left(\gamma-\frac{b^{2} \mathcal{I}_{10}-a^{2} \mathcal{I}_{01}}{a^{2}+b^{2}}\right) V\right] .
$$

From the first term, we have $\triangle(\gamma V)=(\triangle \gamma) V+2 \partial_{i} \gamma \partial_{i} V+\gamma(\triangle V)$ but $(\triangle \gamma) V=0$ from (3.17) while the cross term $\partial_{i} \gamma \partial_{i} V$ contains no $\delta$ function. So, only $\gamma(\triangle V)$ remains. For the second term, $\left[\triangle\left(b^{2} \mathcal{I}_{10}-a^{2} \mathcal{I}_{01}\right)\right] V$ has no $\delta$ function from $(\mathrm{C} .31)$, while $\left(b^{2} \mathcal{I}_{10}-\right.$ $\left.a^{2} \mathcal{I}_{01}\right) \triangle V$ vanishes by (C.30). There is no $\delta$ function from the cross term. So, after all, the only $\delta$-function singularities representing localized codimension- 3 charges are

$$
\begin{aligned}
\triangle K^{3} & =-\frac{Q_{5} \Omega}{2}\left(k+k^{\prime}\right) \gamma(r=0) \Delta V+\triangle K_{(3)}^{3} \\
& = \begin{cases}-\frac{Q_{5} \Omega}{2}\left(k+k^{\prime}\right) \frac{b^{2}}{a^{2}+b^{2}} \Delta V+\triangle K_{(3)}^{3} & (a>b, \text { "southern" }), \\
+\frac{Q_{5} \Omega}{2}\left(k+k^{\prime}\right) \frac{a^{2}}{a^{2}+b^{2}} \Delta V+\triangle K_{(3)}^{3} & (b>a, \text { "northern" }),\end{cases}
\end{aligned}
$$

where in the second equality we used (C.24) for the value of $\gamma(r=0)$. So, we have $-\left(k+k^{\prime}\right) \frac{b^{2}}{a^{2}+b^{2}}$ units of D4 charge sitting at $r=0$ in the "southern" case, and $\left(k+k^{\prime}\right) \frac{a^{2}}{a^{2}+b^{2}}$ units for the "northern" case. The discontinuity as we go from the south to the north will be discussed in section 3.5. 


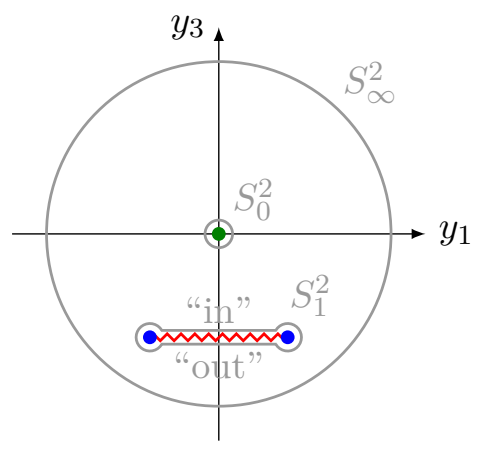

Figure 3. The Gaussian surfaces. The large gray circle represents $S^{2}$ at infinity, which can be deformed into two disconnected surfaces: $S_{0}^{2}$, enclosing the origin, and $S_{1}^{2}$, surrounding the disk $D_{2}$ (red zigzag line) whose boundary is the ring (blue dots). The difference of a quantity $X$ across $D_{2}$ is defined to be $[X]=X_{\text {out }}-X_{\text {in }}$, where "out" and "in" are defined as shown here, for the southern case.

Delocalized charge. Actually, this is not the end of the story. In the current situation where we have a branch cut of the multi-valued function $\gamma$, we can also have delocalized charges.

Recall that the D4 charge is measured by the Gaussian integral inside $\mathbb{R}^{3}$ as

$$
q_{\mathrm{D} 4}=-\frac{1}{4 \pi} \int_{S_{\infty}^{2}} *{ }_{3} d K^{3}
$$

where $S_{\infty}^{2}$ is the sphere at infinity, with its normal pointing outward. We can continuously deform $S_{\infty}^{2}$ into two disconnected pieces one of which encloses $r=0$ and the other of which surrounds the disk $D_{2}$ whose boundary is the ring (see figure 3). Because $K^{3}$ is discontinuous across the disk, there will be non-trivial contribution to the integral there. The only discontinuous part in $d K^{3}$ is

$$
\left.d K^{3}\right|_{\text {discont }}=-\left.\frac{Q_{5} \Omega}{2}\left(k+k^{\prime}\right) d(\gamma V)\right|_{\text {discont }}=-\frac{Q_{5} \Omega}{2}\left(k+k^{\prime}\right) \gamma d V
$$

where we used the fact that $d \gamma=*_{3} d \alpha$ is continuous. Let us denote the discontinuity of $X$ by $[X]=X_{\text {out }}-X_{\text {in }}$, where "out" and "in" mean outside and inside of $D_{2}$, with the orientation derived from that of $S_{\infty}^{2}$, as shown in figure 3 , in the "southern" case $(a>b)$. In the "southern" case, we have $[\gamma]=-1$ and the contribution to (3.33) from the delocalized charge is

$$
\begin{aligned}
q_{\text {deloc }} & =-\frac{1}{4 \pi} \int_{D_{2}}\left[{ }_{3} d K^{3}\right]=\frac{1}{4 \pi} \frac{Q_{5} \Omega}{2}\left(k+k^{\prime}\right) \int_{D_{2}}[\gamma] *_{3} d V \\
& =-\frac{1}{4 \pi} \frac{Q_{5} \Omega}{2}\left(k+k^{\prime}\right) \int_{D_{2}} *_{3} d V=-\frac{1}{4 \pi} \frac{Q_{5} \Omega}{2}\left(k+k^{\prime}\right) \int_{D_{2}} \sin \theta d \theta \wedge d \phi .
\end{aligned}
$$

In the "southern" case $(a>b)$, the $\theta$ integral is from $\theta$ such that $\cos \theta=\frac{c}{\sqrt{c^{2}+R^{2}}}=\frac{b^{2}-a^{2}}{a^{2}+b^{2}}$ to $\theta=\pi$. So, evaluating the integral, we find

$$
q_{\text {deloc }}= \begin{cases}+\frac{Q_{5} \Omega}{2}\left(k+k^{\prime}\right) \frac{b^{2}}{a^{2}+b^{2}} & (a>b, \text { "southern" }) \\ -\frac{Q_{5} \Omega}{2}\left(k+k^{\prime}\right) \frac{a^{2}}{a^{2}+b^{2}} & (b>a, \text { "northern" })\end{cases}
$$


We also included the expression for the "northern" case (in which $[\gamma]=+1$ ). The discontinuity as we go from the south to the north will be discussed in section 3.5. The sum of this delocalized charge and the charge localized at $r=0$ in (3.32) is zero.

The expression for $\boldsymbol{K}^{\mathbf{3}}$. The codimension-3 part $K_{(3)}^{3}$ is chosen so that the ring carries $k$ units of D4 charge (including the localized and delocalized charges) in the southern case:

$$
K_{(3)}^{3}=\frac{Q_{5} \Omega}{2} \frac{a^{2} k-b^{2} k^{\prime}}{a^{2}+b^{2}}(H-V) .
$$

Actually, here we added a term proportional to $V$, which can be thought of as the "gauge transformation" (2.6). We will see that this choice agrees with the $6 \mathrm{~d}$ result in the next section. For continuity, we must take the same $K_{(3)}^{3}$ also for the northern case.

So, the final expression for $K^{3}$ is

$$
K^{3}=\frac{Q_{5} \Omega}{2}\left[-\left(k+k^{\prime}\right)\left(\gamma-\frac{b^{2} \mathcal{I}_{10}-a^{2} \mathcal{I}_{01}}{a^{2}+b^{2}}\right) V+\frac{a^{2} k-b^{2} k^{\prime}}{a^{2}+b^{2}}(H-V)\right] .
$$

\subsection{Integrability and no-CTC conditions}

Having determined $K^{3}$, we can find the remaining harmonic function $M$. First, It must contain $\gamma$ so that $\mu$ (2.38) is single-valued. Furthermore, it must satisfy the integrability condition derived from (2.39),

$$
0=V \triangle M-M \triangle V-\frac{1}{2} \triangle K^{3} \quad \text { (no } \delta \text { function). }
$$

Imposing these conditions, it is straightforward to show that $M$ is given by:

$$
M=\frac{Q_{5} \Omega}{4}\left[\left(k+k^{\prime}\right) \gamma+\frac{1}{4}\left(k a^{2}-k^{\prime} b^{2}\right) H\right] .
$$

The 1-form $\omega$ that satisfies the equation (2.39) is

$$
\omega=-\frac{Q_{5} \Omega}{2}\left(k \mathcal{I}_{10} \cos ^{2} \frac{\theta}{2}+k^{\prime} \mathcal{I}_{01} \sin ^{2} \frac{\theta}{2}\right) d \phi .
$$

The modulus $\tau^{3}$ (see (2.11)) is found to be

$$
\tau^{3}=-\left(k+k^{\prime}\right) \gamma+(\text { single-valued })
$$

which has the monodromy $\tau^{3} \rightarrow \tau^{3}-\left(k+k^{\prime}\right)$ due to the $-\left(k+k^{\prime}\right)$ NS5 $(4567 \lambda)$ branes. This monodromy can be represented by an $\mathrm{SL}(2, \mathbb{Z})$ transformation

$$
\mathcal{M}=\left(\begin{array}{cc}
1 & -\left(k+k^{\prime}\right) \\
0 & 1
\end{array}\right)
$$

At this point, the solution contains $Q_{1}, Q_{5}, a, b, k, k^{\prime}$ as independent parameters. In the Lunin-Mathur geometry, they are going to be related by the relation $(2.22 \mathrm{c})$; for example, in the south-pole limit it is given by (2.27b). In the current framework of harmonic solutions in $4 \mathrm{~d} / 5 \mathrm{~d}$, such a relation can be derived by studying the no-CTC condition. 
Let us focus on the $5 \mathrm{~d}$ part of the $11 \mathrm{~d}$ metric (2.1). If we focus on the $\phi, \psi$ part, we have

$$
Z^{2 / 3} d s_{5}^{2} \supset-\left[\mu\left(d \psi+A_{\phi} d \phi\right)+\omega_{\phi} d \phi\right]^{2}+Z\left[V^{-1}\left(d \psi+A_{\phi} d \phi\right)^{2}+V r^{2} \sin ^{2} \theta d \phi^{2}\right]
$$

where we used (2.5c). Near the ring, $u \sim \infty$, the quantities appearing here behave as (see (C.29), (C.30))

$$
\begin{array}{rlrl}
\omega_{\phi} & \sim \frac{Q_{5} \Omega}{2} \frac{a b}{a^{2}+b^{2}}\left(k+k^{\prime}\right) \frac{\log u}{\pi}, & \mu & \sim \frac{Q_{5} \Omega}{4}\left(k \frac{a}{b}-k^{\prime} \frac{b}{a}\right) \frac{\log u}{\pi}, \\
A_{\phi} & \sim \frac{2 b^{2}}{a^{2}+b^{2}}, \quad V \sim \frac{4}{a^{2}+b^{2}}, & Z \sim \frac{Q_{1} Q_{5}}{4 \pi^{2} a^{2} b^{2}} \log ^{2} u, \quad g_{\phi \phi}=r^{2} \sin ^{2} \theta \sim \frac{a^{2} b^{2}}{4} .
\end{array}
$$

By diagonalizing the metric (3.44) and requiring that there is no CTC, namely the eigenvalues are non-negative, we find the condition

$$
Q_{1} \geq \Omega^{2}\left(a^{2} k^{2}+b^{2} k^{2}\right) Q_{5}
$$

In supertube configurations, genuine microstates saturate such non-CTC inequalities [45]. Because Lunin-Mathur geometries are genuine microstates, we must require

$$
Q_{1}=\Omega^{2}\left(a^{2} k^{2}+b^{2} k^{2}\right) Q_{5}
$$

This is what we used in (1.5).

\subsection{Comments}

Thus far, in this section, we have demonstrated the phenomenon of charge transfer, which is summarized in figure 1. The D4 charge has two parts, the one localized at the location of the ring, and the other distributed over the branch cut inside the ring. As we move from the south pole to the north pole, the cut crosses the D6 center at $r=0$ and, at that point, some of the D4 charge gets transferred to the D6 center. As the result, the ring has different D4 charges in the initial and final states.

This can be understood in terms of a "gauge transformation". Let us note that, if we apply the gauge transformation (2.6) with $c^{1}=c^{2}=0, c^{3}=-n Q_{5}\left(k^{\prime}+k\right) \Omega / 2$ to the harmonic functions (3.38), (3.40), we obtain

$$
\begin{aligned}
& K^{3}=\frac{Q_{5} \Omega}{2}\left[-\left(k+k^{\prime}\right)\left(\gamma+n-\frac{b^{2} \mathcal{I}_{10}-a^{2} \mathcal{I}_{01}}{a^{2}+b^{2}}\right) V+\frac{a^{2} k-b^{2} k^{\prime}}{a^{2}+b^{2}}(H-V)\right], \\
& M=\frac{Q_{5} \Omega}{4}\left[\left(k+k^{\prime}\right)(\gamma+n)+\frac{1}{4}\left(k a^{2}-k^{\prime} b^{2}\right) H\right] .
\end{aligned}
$$

Let us consider the process of starting from the south pole limit with $n=0$ and moving over to the north pole. In the "southern" case $a>b$, the branch cut is at $y_{3}=\left(b^{2}-a^{2}\right) / 4<0$ and at the position of the $r=0$ center, we have $0<\gamma<1 / 2$. From (3.32), we see that the D4 charge of the $r=0$ center is $-\frac{Q_{5} \Omega}{2}\left(k+k^{\prime}\right) \frac{b^{2}}{a^{2}+b^{2}}$. If we increase $b$ and go over the to the "northern" case $a<b$, the $r=0$ pole has gone through the ring and now $\gamma>1 / 2$ there. If we want to bring it back to the $-1 / 2<\gamma<1 / 2$ branch which can be connected 
to infinity (note that $\gamma \rightarrow 0$ at infinity), we must do a gauge transformation with $n=-1$ so that $-1 / 2<\gamma<1 / 2$ there. This is how we get $\frac{Q_{5} \Omega}{2}\left(k+k^{\prime}\right) \frac{a^{2}}{a^{2}+b^{2}}$ in (3.32) as the D4 charge of the $r=0$ center. Namely, the "jump" in the D4 charge happens because of the gauge transformation needed to bring the $r=0$ pole, which has gone to a different gauge by going through the ring, back into the original gauge.

In the current note, we only discussed the "helical" profile (3.1), but we could consider any $4 \mathrm{~d}$ profile and its reduction to $\mathbb{R}^{3}$. In $\mathbb{R}^{3}$, it will give an NS5-brane along some arbitrary curve $\mathcal{C}$, with $\mathrm{D} 4$ charge density which can vary along $\mathcal{C}$. If we define a 1 -form $\alpha$ as in (3.15) (now by an integration along $\mathcal{C}$ ) and the dual scalar $\gamma$ by $d \gamma=*_{3} d \alpha$, the construction of $K^{3}$ goes just as in (3.25)-(3.27), although explicit expressions will be harder to obtain.

\section{Deriving harmonic functions from $6 \mathrm{~d}$}

In the previous section, we derived the harmonic functions with codimension- 2 sources that represent a Lunin-Mathur geometry in $6 \mathrm{~d}$, based on the information about what charges must be present (bottom-up). Here, we confirm that these harmonic functions are correct, starting from $6 \mathrm{~d}$ (top-down).

The various functions of the Lunin-Mathur geometry (2.22) computed for the profile (3.1) are given by

$$
\begin{aligned}
& Z_{1}=\frac{Q_{1}}{4} H, \quad Z_{2}=\frac{Q_{2}}{4} H, \quad Q_{1}=\Omega^{2}\left(k^{2} a^{2}+k^{\prime 2} b^{2}\right) Q_{5}, \\
& \mathbf{A}_{\widetilde{\phi}}=-\frac{Q_{5} \Omega k}{2} \mathcal{I}_{10}, \quad \mathbf{A}_{\widetilde{\psi}}=+\frac{Q_{5} \Omega k^{\prime}}{2} \mathcal{I}_{01}, \\
& \mathbf{B}_{\widetilde{\phi}}=\frac{Q_{5} \Omega k^{\prime}}{2}\left(-\mathcal{I}_{01}+\frac{1}{2} b^{2} H-2 \gamma\right), \quad \mathbf{B}_{\widetilde{\psi}}=\frac{Q_{5} \Omega k}{2}\left(\mathcal{I}_{10}-\frac{1}{2} a^{2} H-2 \gamma\right) .
\end{aligned}
$$

where $\widetilde{\phi}, \widetilde{\psi}$ were defined in (2.29), and other components of $\mathbf{A}, \mathbf{B}$ vanish. The computation is straightforward, if complicated. The only thing is that we must smear over the $\psi$ direction. For some detail see (C.4). The functions $Z_{1}, Z_{2}$ are the same as the one found from the $4 \mathrm{~d}$ viewpoint in (3.22). Also, in $6 \mathrm{~d}$, the relation (3.47) is automatic. If we set $b \rightarrow 0$, (4.1) reduces to $(2.27)$.

We can find what harmonic functions these reduce to, using the results in section 2.3. We can decompose the 1-forms $\mathbf{A}, \mathbf{B}$ as

$$
\begin{aligned}
& \mathbf{A}=\frac{\mathbf{A}_{\widetilde{\phi}}+\mathbf{A}_{\widetilde{\psi}}}{2}(d \psi+A)+\left(-\mathbf{A}_{\widetilde{\phi}} \cos ^{2} \frac{\theta}{2}+\mathbf{A}_{\widetilde{\psi}} \sin ^{2} \frac{\theta}{2}\right) d \phi, \\
& \mathbf{B}=\frac{\mathbf{B}_{\widetilde{\phi}}+\mathbf{B}_{\widetilde{\psi}}}{2}(d \psi+A)+\left(-\mathbf{B}_{\widetilde{\phi}} \cos ^{2} \frac{\theta}{2}+\mathbf{B}_{\widetilde{\psi}} \sin ^{2} \frac{\theta}{2}\right) d \phi .
\end{aligned}
$$

Using the identification (2.33), we find

$$
\begin{array}{rlrl}
M & =-\frac{1}{4}\left(\mathbf{A}_{\widetilde{\phi}}+\mathbf{A}_{\widetilde{\psi}}+\mathbf{B}_{\widetilde{\phi}}+\mathbf{B}_{\widetilde{\psi}}\right), & K^{3}=\frac{V}{2}\left(-\mathbf{A}_{\widetilde{\phi}}-\mathbf{A}_{\widetilde{\psi}}+\mathbf{B}_{\widetilde{\phi}}+\mathbf{B}_{\widetilde{\psi}}\right), \\
\mu & =-\frac{1}{2}\left(\mathbf{A}_{\widetilde{\phi}}+\mathbf{A}_{\widetilde{\psi}}\right), & \omega & =\left(\mathbf{A}_{\widetilde{\phi}} \cos ^{2} \frac{\theta}{2}-\mathbf{A}_{\widetilde{\psi}} \sin ^{2} \frac{\theta}{2}\right) d \phi, \\
\xi^{3} & =\left[\left(\mathbf{A}_{\widetilde{\phi}}-\mathbf{B}_{\widetilde{\phi}}\right) \cos ^{2} \frac{\theta}{2}+\left(-\mathbf{A}_{\widetilde{\psi}}+\mathbf{B}_{\widetilde{\psi}}\right) \sin ^{2} \frac{\theta}{2}\right] d \phi .
\end{array}
$$


It is not difficult to show that these reproduce the harmonic functions (3.38), (3.40) in the previous section, using the formula (C.25).

The reader may think that, to find harmonic functions, it is much easier to start with the Lunin-Mathur geometry in $6 \mathrm{~d}$ and go down to $4 \mathrm{~d} / 5 \mathrm{~d}$ as above ("top-down"), rather than going from $4 \mathrm{~d} / 5 \mathrm{~d}$ to $6 \mathrm{~d}$ as we did in section 3 ("bottom-up"). However, a technical point is that, even if we know $\mathbf{A}$, the relation (2.33a) only gives us the combination $\mu=M+K^{3} /(2 V)$, and not $M$ and $V$ separately. One could use the harmonicity of $K^{3}, M$ to disentangle them from each other, but that is far from simple. If we also know $\mathbf{B}$, it is easy to find $K^{3}, M$ from the two relations (2.33), but finding $\mathbf{B}$ from the duality relation (2.22b) is not simple either. We found the expression for $\mathbf{B}$ in (4.1) by first finding $K^{3}, M$ and then going back up to $6 \mathrm{~d}$ via $(2.33 \mathrm{~b})$ and $(4.2 \mathrm{~b})$.

\section{Dual CFT perspective and spectral flow}

\subsection{Dual CFT states}

The CFT dual of general Lunin-Mathur geometries are known [39, 42, 46, 47]. Here we discuss the dual for the special case of the helical profile, focusing on its symmetry.

The holographic dual of the D1-D5 system is a 2d SCFT called the D1-D5 CFT, which is an orbifold CFT with target space $\operatorname{Sym}^{N}\left(T^{4}\right), N=N_{1} N_{5} \cdot{ }^{11}$ The CFT has $\mathrm{SU}(2)_{L} \times \mathrm{SU}(2)_{R}$ R-symmetry with generators $J_{L}^{i}, J_{R}^{i}$ and the states in the CFT have Rcharges $\left(J_{L}^{3}, J_{R}^{3}\right)=\left(j_{L}, j_{R}\right)$. For more detail of the D1-D5 CFT, see [48, 49]. Our notation here follows [50].

The states of the orbifold CFT can be constructed multiplying "strands" of various length together so that the total length is $N$. The CFT state that is dual to the circular profile (1.1) is

$$
\left[|++\rangle_{k}\right]^{N / k}, \quad N=N_{1} N_{5}
$$

Here $|++\rangle_{k}$ is a strand of length $k$, with a Ramond-Ramond ground state with R-charge $\left(j_{L}, j_{R}\right)=\left(\frac{1}{2}, \frac{1}{2}\right)$ on it, denoted by " ++ ". This state is an eigenstate of $J_{L}^{3}, J_{R}^{3}$. The dual bulk statement is that the supergravity solution preserves the corresponding $\mathrm{U}(1)_{L} \times \mathrm{U}(1)_{R}$ symmetry. More precisely, $J_{\widetilde{\phi}}=J_{L}^{3}+J_{R}^{3}$ and $J_{\widetilde{\psi}}=J_{L}^{3}-J_{R}^{3}$ generate rotations in the 1-2 and 3-4 planes under which the circular profile (2.25) is invariant.

On the other hand, the state dual to the helical profile (1.4) is a coherent sum of RR ground states [39, 42, 46, 47],

$$
\sum_{N_{++}, N_{-+}}^{\prime}\left[A_{++}|++\rangle_{k}\right]^{N_{++}}\left[A_{-+}|-+\rangle_{k^{\prime}}\right]^{N_{-+}},
$$

where the sum is restricted to $\left(N_{++}, N_{-+}\right)$with $k N_{++}+k^{\prime} N_{-+}=N$, and the parameters $A_{++}, A_{-+}$are related to the bulk quantities $a, b$ via

$$
\left|A_{++}\right|^{2}=\frac{R_{y}^{2} N}{Q_{1} Q_{5}} k^{2} a^{2}, \quad\left|A_{-+}\right|^{2}=\frac{R_{y}^{2} N}{Q_{1} Q_{5}} k^{\prime 2} b^{2} .
$$

\footnotetext{
${ }^{11}$ More precisely the target space is a deformation of this but we will assume that the target space is this orbifold.
} 
The sum (5.2) is dominated by the following term:

$$
k \bar{N}_{++}=\left|A_{++}\right|^{2}, \quad k^{\prime} \bar{N}_{-+}=\left|A_{-+}\right|^{2} .
$$

The state (5.2) is not an eigenstate of $J_{L}^{3}$ and $J_{R}^{3}$ separately, but it is an eigenstate of the combination $\left(k-k^{\prime}\right) J_{L}^{3}+\left(k+k^{\prime}\right) J_{R}^{3}=k J_{\widetilde{\phi}}-k^{\prime} J_{\widetilde{\psi}}{ }^{12}$ In the bulk, this is nothing but the linear combination of $\widetilde{\phi}$ and $\widetilde{\psi}$ directions under which the helical profile is invariant. Once we project the profile to $\mathbb{R}^{3}$, this structure becomes invisible because of smearing; namely, the $4 \mathrm{~d} / 5 \mathrm{~d}$ configuration is symmetric under both $\phi$ and $\psi$ translations, although this is an artifact of smearing.

As we keep increasing $b$, we end up with the $a=0, b>0$ state

$$
\left[|-+\rangle_{k^{\prime}}\right]^{N / k^{\prime}}
$$

which corresponds to the "north pole" limit. This is again symmetric under $\mathrm{U}(1)_{L} \times \mathrm{U}(1)_{R}$.

\subsection{Spectral flow}

Being an $\mathcal{N}=(4,4)$ SCFT, the D1-D5 CFT has spectral flow symmetry [51] which maps a state with $L_{0}=h, J_{L}^{3}=j_{L}$ (we take $L_{0}=0$ for Ramond ground states) on a strand of length $k$ to a state with $L_{0}=h^{\prime}, J_{L}^{3}=j_{L}^{\prime}$ as

$$
h^{\prime}=h+2 n j_{L}+k n^{2}, \quad j_{L}^{\prime}=j_{L}+k n .
$$

For $n \in \mathbb{Z}$, this maps states in the Ramond sector into states in the Ramond sector.

Let us denote the Ramond state obtained by spectral flowing $| \pm+\rangle_{k}$ by $n$ by

$$
| \pm+\rangle_{k, n} \quad \text { with } \quad h= \pm n+k n^{2}, \quad j_{L}= \pm \frac{1}{2}+k n .
$$

By spectral flowing the state (5.1), we obtain

$$
\left[| \pm+\rangle_{k, n}\right]^{N / k}
$$

If we spectral flow the state (5.2), we obtain the coherent sum

$$
\sum_{N_{++}, N_{-+}}^{\prime}\left[A_{++}|++\rangle_{k, n}\right]^{N_{++}}\left[A_{-+}|-+\rangle_{k^{\prime}, n}\right]^{N_{-+}},
$$

which has charges

$$
\begin{aligned}
h & =\left(n+k n^{2}\right) \bar{N}_{++}+\left(-n+k^{\prime} n^{2}\right) \bar{N}_{-+}, \\
j_{L} & =\left(\frac{1}{2}+k n\right) \bar{N}_{++}+\left(-\frac{1}{2}+k^{\prime} n\right) \bar{N}_{-+}, \\
j_{R} & =\frac{1}{2}\left(\bar{N}_{++}+\bar{N}_{-+}\right) .
\end{aligned}
$$

\footnotetext{
${ }^{12}$ One exceptional case is $k=k^{\prime}$. In this case, making a non-vanishing can be thought of merely as an $\mathrm{SU}(2)_{L}$ rotation of the original state (5.1). Indeed, one can show that (5.1) is an eigenstate of $J_{R}^{3}$ and a certain linear combination of $J_{L}^{i}, i=1,2,3$.
} 
In the bulk, the spectral flow transformation is realized by the following transformation of the harmonic functions [52]

$$
\begin{aligned}
& \tilde{V}=V+\gamma^{3} K^{3}, \quad \tilde{K}^{1}=K^{1}-\gamma^{3} L_{2}, \quad \tilde{K}^{2}=K^{2}-\gamma^{3} L_{1}, \quad \tilde{K}^{3}=K^{3}, \\
& \tilde{L}_{3}=L_{3}-2 \gamma^{3} M, \quad \tilde{L}_{2}=L_{2}, \quad \tilde{L}{ }_{1}=L_{1}, \quad \tilde{M}=M, \quad \tilde{\omega}=\omega,
\end{aligned}
$$

under which the moduli $\tau^{I}$ defined in (2.11) transform as

$$
\tau^{1} \rightarrow \tau^{1}, \quad \tau^{2} \rightarrow \tau^{2}, \quad \tau^{3} \rightarrow \frac{\tau^{3}}{\frac{Q_{5} \Omega}{2} \gamma^{3} \tau^{3}+1}
$$

Namely, it is an $\operatorname{SL}(2, \mathbb{Z})$ duality transformation of the modulus of $T_{89}^{2} \cdot{ }^{13}$

Let us study the bulk dual of the spectral flowed states above. First, applying (5.11) with $\gamma^{3}=-\frac{2 n}{Q_{5} \Omega}$ to the codimension-3 harmonic functions (1.2) and doing gauge transformation (2.6) with $c^{1}=-\frac{n}{2 \Omega}, c^{2}=-\frac{n Q_{1}}{2 Q_{5} \Omega}, c^{3}=0$ so that $K^{I}$ are $\mathcal{O}\left(1 / r^{2}\right)$ at infinity, we find the dual of (5.8):

$$
\begin{array}{rlrl}
\tilde{V} & =\frac{1+k n}{r}-\frac{k n}{\Sigma}, & \tilde{K}^{3}=\frac{Q_{5} \Omega k}{2}\left(\frac{1}{\Sigma}-\frac{1}{r}\right), \\
\tilde{K}^{1}=\frac{n(k n+1)}{2 \Omega}\left(\frac{1}{\Sigma}-\frac{1}{r}\right), & \tilde{K}^{2}=\frac{Q_{1} n(k n+1)}{2 Q_{5} \Omega}\left(\frac{1}{\Sigma}-\frac{1}{r}\right), \\
\tilde{L}_{1}=\frac{Q_{1}}{4}\left(\frac{k n+1}{\Sigma}-\frac{k n}{r}\right), & \tilde{L}_{2}=\frac{Q_{5}}{4}\left(\frac{k n+1}{\Sigma}-\frac{k n}{r}\right), \\
\tilde{L}_{3}=1+\frac{Q_{1} n(k n+1)}{4 Q_{5} \Omega^{2} k}\left(\frac{k n+1}{\Sigma}-\frac{k n}{r}\right), & \tilde{M} & =\frac{Q_{1}}{16 \Omega k}\left(\frac{(k n+1)^{2}}{\Sigma}-\frac{(k n)^{2}}{r}\right) .
\end{array}
$$

Next, if we apply the same bulk spectral flow and gauge transformations to the codimension2 harmonic functions given by (3.22), (3.38) and (3.40), we obtain

$$
\begin{aligned}
\tilde{V} & =\frac{1}{r}-\frac{2 n}{Q_{5} \Omega} K^{3}, \quad \tilde{K}^{1}=-\frac{n}{2 \Omega} \frac{1}{r}+\frac{n}{2 \Omega} H+\frac{n^{2}}{Q_{5} \Omega^{2}} K^{3}, & \tilde{K}^{2}=\frac{Q_{1}}{Q_{5}} \tilde{K}^{1}, \\
\tilde{K}^{3} & =K^{3}, \quad \tilde{L}_{1}=\frac{Q_{1}}{4} H+\frac{n Q_{1}}{2 Q_{5} \Omega} K^{3}, & \tilde{L}_{2}=\frac{Q_{5}}{4} H+\frac{n}{2 \Omega} K^{3}, \\
\tilde{L}_{3}=1-\frac{n^{2} Q_{1}}{4 Q_{5} \Omega^{2}} \frac{1}{r}+\frac{n^{2} Q_{1}}{2 Q_{5} \Omega^{2}} H+\frac{n^{3} Q_{1}}{2 Q_{5}^{2} \Omega^{3}} K^{3}+\frac{4 n}{Q_{5} \Omega} M, & & \\
\tilde{M} & =M+\frac{n Q_{1}}{8 \Omega} H+\frac{n^{2} Q_{1}}{8 Q_{5} \Omega^{2}} K^{3} &
\end{aligned}
$$

where $K^{3}, M$ are the ones given in (3.38), (3.40). This must be the bulk dual of the CFT state (5.9). From the $1 / r$ fall-off of $\tilde{L}_{3}$ and $\tilde{M}$, we can confirm the charges (5.10). For

\footnotetext{
${ }^{13}$ Generalization of this to a three-parameter family of transformations with parameters $\gamma^{I}, I=1,2,3$ gives an $\operatorname{SL}(2, \mathbb{Z})$ duality transformation for $\tau^{I}[52]$.
} 
example, the large $r$ expansion of $\tilde{L}_{3}$ is

$$
\begin{aligned}
\tilde{L}_{3} & \sim\left[-\frac{n^{2} Q_{1}}{4 Q_{5} \Omega^{2}}+\frac{n^{2} Q_{1}}{2 Q_{5} \Omega^{2}}+\frac{4 n}{Q_{5} \Omega} \frac{Q_{5} \Omega}{4} \frac{1}{4}\left(k a^{2}-k^{\prime} b^{2}\right)\right] \frac{1}{r} \\
& =\frac{1}{4 r}\left[\left(n+k n^{2}\right) k a^{2}+\left(-n+k^{\prime} n^{2}\right) k^{\prime} b^{2}\right] \equiv \frac{Q_{p}}{4 r},
\end{aligned}
$$

where we used (3.47). Using (5.3) and (5.4), and using the relation between $Q_{p}$ and the quantized momentum $N_{p}$,

$$
Q_{p}=\frac{g_{s}^{2} \alpha^{4}}{R_{y}^{2} v_{4}} N_{p}=\frac{Q_{1} Q_{5}}{R_{y}^{2} N} N_{p},
$$

it is easy to show that this reproduces the CFT result (5.10a).

So, the harmonic solution (5.14) provides the supergravity description of the CFT state (5.9). What is interesting is that, because $\tilde{V}$ contains the monodromic $K^{3}$ that can transfer charge, the solution interpolates between Gibbons-Hawking spaces with different Taub-NUT charges.

$$
\tilde{V}=\frac{1+k n}{r}-\frac{k n}{\Sigma} \quad \rightarrow \quad \tilde{V}=\frac{1-k^{\prime} n}{r}+\frac{k^{\prime} n}{\Sigma^{\prime}},
$$

where $\Sigma^{\prime}=|\mathbf{y}-\widetilde{\mathbf{b}}|, \widetilde{\mathbf{b}}=(0,0, \widetilde{b}), \widetilde{b}=b^{2} / 4$. In particular, if $k=k^{\prime}=1, n=-m$, this connects the two configurations:

$$
\tilde{V}=\frac{1-m}{r}+\frac{m}{\Sigma} \rightarrow \tilde{V}=\frac{1+m}{r}-\frac{m}{\Sigma^{\prime}}=\frac{1-(m+1)}{\Sigma^{\prime}}+\frac{m+1}{r} .
$$

On the right hand side, by reinterpreting $\Sigma^{\prime} \rightarrow r, r \rightarrow \Sigma$, we can repeat the same process now with $m \rightarrow m+1$. Doing this over again, we can reach any $m$. See figure 4 for a graphical explanation of this process. In CFT, the process (5.18) corresponds to the interpolation

$$
\left[|++\rangle_{1,-m}\right]^{N} \rightarrow\left[|-+\rangle_{1,-m}\right]^{N}=\left[|++\rangle_{1,-(m+1)}\right]^{N},
$$

where in the last expression we used the fact that $|-+\rangle_{1,-m}=\left[|++\rangle_{1,-(m+1)}\right]$. So, by starting from $\left[|++\rangle_{1}\right]^{N}=\left[|++\rangle_{1,0}\right]^{N}$ and repeatedly doing this interpolation, we can get to $\left[|++\rangle_{1,-m}\right]^{N}$ with any $m$; see figure 5 for a description of this spectral flow on the $J_{L}^{3}-L_{0}$ plane.

From (3.43) and (5.12), we see that the modulus $\tau^{3}$ now has the $\operatorname{SL}(2, \mathbb{Z})$ monodromy

$$
\tilde{\mathcal{M}}=\left(\begin{array}{cc}
1 & -\left(k+k^{\prime}\right) \\
-n & 1+n\left(k+k^{\prime}\right)
\end{array}\right) .
$$

This is no longer the monodromy of NS5(4567C)-branes but the "exotic brane" $5_{2}^{2}(4567 \mathcal{C}, 89)[43]$ has mixed in. By following the duality transformation to the D1-D5 frame, we see that this is mapped as

$$
5_{2}^{2}(4567 \mathcal{C}, 89) \stackrel{\text { dualize }}{\longrightarrow} \operatorname{KKM}(6789 \mathcal{C}, \psi) .
$$

So, in addition to the KKMs in (2.34), we have new a kind of KKM dipole, but the solution is purely geometric. This is as it should be, because the spectral flow transformation in the D1-D5 frame is nothing but mixing the coordinates $y$ and $\psi$; part of the KKM in the second line of $(2.34)$ got transformed into $\operatorname{KKM}(6789 \mathcal{C}, \psi)$ above. 


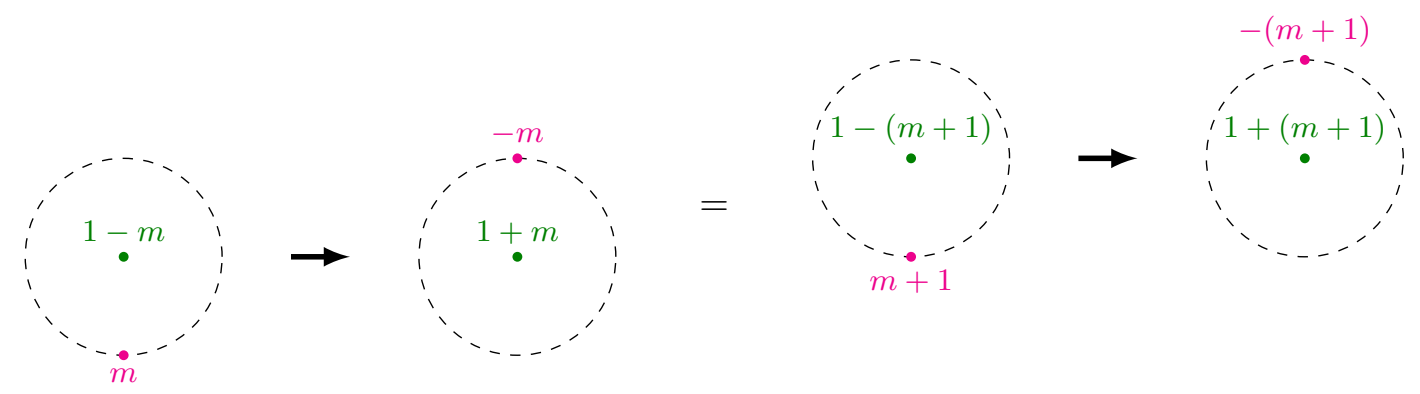

Figure 4. Repeated interpolations in the bulk.

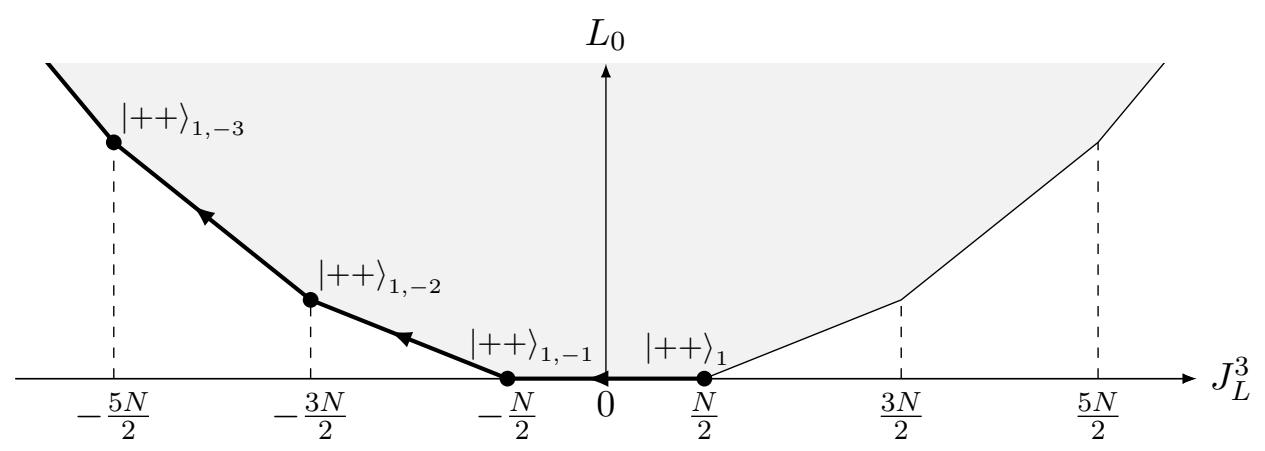

Figure 5. Repeated interpolations in CFT to reach $\left[|++\rangle_{1,-m}\right]^{N}$ with arbitrary $m$.

\subsection{Fractional spectral flow}

For general CFT states, the spectral flow is defined only for $n \in \mathbb{Z}$. However, for the state $| \pm+\rangle_{k}$, the fractional spectral flow by $n=\frac{s}{k}, s \in \mathbb{Z}$,

$$
| \pm+\rangle_{k, \frac{s}{k}}: \quad h=\frac{s(s \pm 1)}{k}, \quad j_{L}= \pm \frac{1}{2}+s
$$

is also a valid state [53] if

$$
h=\frac{s(s \pm 1)}{k} \in \mathbb{Z}, \quad \text { or } \quad n(n k \pm 1) \in \mathbb{Z} .
$$

Indeed, the dual bulk solution (5.13) is known to make sense [53] if this condition (5.23) is met, and represents the most general 2-center codimension-3 harmonic solution.

The interpolating CFT state (5.9) must also be a valid state if both the states $|++\rangle_{k, n}$ and $|-+\rangle_{k^{\prime}, n}$ satisfy the quantization condition, namely,

$$
n(n k+1) \in \mathbb{Z} \quad \text { and } \quad n\left(n k^{\prime}-1\right) \in \mathbb{Z} .
$$

For example, for $m, m^{\prime} \in \mathbb{Z}_{>0}$, the following states with $n=1 / 2$ :

$$
\begin{aligned}
& |++\rangle_{4 m+2, \frac{1}{2}}, \quad h=m+1 \in \mathbb{Z}, \quad j_{L}=2 m+\frac{3}{2}, \\
& |-+\rangle_{4 m^{\prime}-2, \frac{1}{2}}, \quad h=m^{\prime}-1 \in \mathbb{Z}, \quad j_{L}=2 m^{\prime}-\frac{3}{2}
\end{aligned}
$$

satisfy the quantization condition and the interpolation will be an allowed state. The dual geometry is still given by (5.14). 


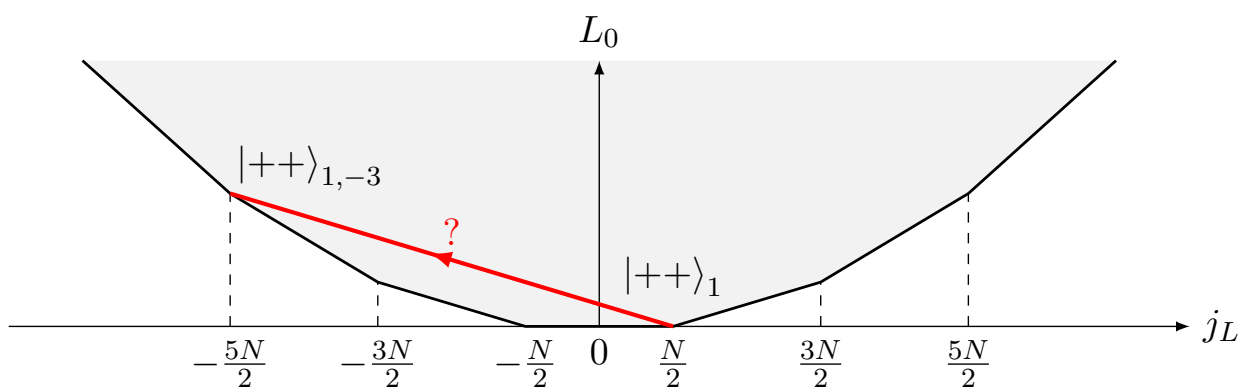

Figure 6. An example of interpolation between $\left[|++\rangle_{k, n}\right]^{N / k}$ and $\left[|++\rangle_{k^{\prime}, n^{\prime}}\right]^{N / k^{\prime}}$ with $n \neq n^{\prime}$.

\section{Discussions}

In this note, we studied the Lunin-Mathur geometry interpolating the states

$$
\left[|++\rangle_{k}\right]^{N / k} \text { and }\left[|-+\rangle_{k^{\prime}}\right]^{N / k^{\prime}}
$$

in the framework of harmonic solutions in $4 \mathrm{~d} / 5 \mathrm{~d}$. Although the geometries dual to (6.1) are codimension- 3 solutions, the interpolating solution is of codimension two, because of the puffed-out NS5-branes lying along a curve $\mathcal{C}$. We discussed how to construct the associated harmonic functions with a monodromy around $\mathcal{C}$, based on the data about the charges and the puffed-out dipole charge. The interpolating solution exhibits some interesting features, such as some of the D4-charge being delocalized, and some of the D4-charge getting transferred from the supertube center to the Taub-NUT center as the interpolation proceeds. We also discussed the spectral flow of this entire process, which interpolates between the states

$$
\left[|++\rangle_{k, n}\right]^{N / k} \text { and } \quad\left[|-+\rangle_{k^{\prime}, n}\right]^{N / k^{\prime}}
$$

This solution is valid even if $n$ is not an integer, as long as certain quantization conditions are met; in that case, the interpolating solution connects the 2-center solutions found in [53].

A natural question [54] is how to interpolate between the states

$$
\left[|++\rangle_{k, n}\right]^{N / k} \text { and }\left[|-+\rangle_{k^{\prime}, n^{\prime}}\right]^{N / k^{\prime}}
$$

where $n \neq n^{\prime}$. For definiteness, let us set $k=k^{\prime}=1, n=0$, and $n^{\prime} \rightarrow n+1$. Then the interpolation in question is between

$$
\left[|++\rangle_{1}\right]^{N} \quad \text { and } \quad\left[|-+\rangle_{1, n+1}\right]^{N}=\left[|++\rangle_{1, n}\right]^{N} .
$$

See figure 6 for an example. In CFT, it is easy to write down the interpolating state, as in (5.9):

$$
\sum_{N_{++}, N_{-+}}^{\prime}\left[A_{1}|++\rangle_{1}\right]^{N_{++}}\left[A_{n}|++\rangle_{1, n}\right]^{N_{-+}}
$$


Just as (5.9), this is an eigenstate of $J_{R}$ and a certain linear combination of $J_{L}$ and $J_{R}$. This suggests that the bulk dual has a helical structure similar to the helical Lunin-Mathur profile that we studied. When reduced to $\mathbb{R}^{3}$, it is likely to be described by a solution involving codimension-2 sources similar to the one studied in this note. Constructing such solutions would be very interesting for understanding black hole microstates, because the state $|++\rangle_{k, n}$ in the background of $|++\rangle_{1}$ is a stringy state in $\mathrm{AdS}_{3} \times S^{3}$ [55-57], which is a key for understanding the fractional and higher modes responsible for the entropy. The technique developed in this note should be useful for such construction. Note, however, that the interpolating states in CFT such as (6.5) are non-BPS when perturbed away from the orbifold point $[54,57]$. Our harmonic function techniques will be directly useful only if such states are actually BPS in supergravity. Such a possibility is not outrageous, because of the observed correspondence between BPS states in the CFT at the orbifold point and multi-center solutions in supergravity [58].

Relatedly, in [20], the moduli space of the multi-center codimension-3 solutions for small numbers of D6- and D2-branes was investigated. They found that, for one D6- and three D2-branes, the number of solutions agrees with the prediction from quiver quantum mechanics, although for more D6-branes the number of multi-center solutions is less than the prediction. It would be interesting to explore the moduli space of multi-center solutions including codimension-2 sources. The results in this note should be useful for such exploration.

\section{Acknowledgments}

I would like to thank CEA Saclay for their (online) hospitality in the "Black-Hole Microstructure I \& II" workshops. This work was supported in part by MEXT KAKENHI Grant Numbers 17H06357 and 17H06359.

\section{A Duality transformation}

Here we discuss how to map the IIB configuration in (2.34) in the D1-D5 frame into the IIA configuration (2.35).

We start with (2.34):

$$
\mathrm{D} 1(y)+\mathrm{D} 5(y 6789) \stackrel{\text { puff out }}{\longrightarrow}\left\{\begin{array}{l}
\operatorname{KKM}(6789 \psi, y)+\mathrm{P}(\psi) \\
\operatorname{KKM}(6789 \mathcal{C}, y)+\mathrm{P}(\mathcal{C})
\end{array}\right.
$$

After T-duality along $y 67$, this is mapped into

$$
\mathrm{D} 2(67)+\mathrm{D} 2(89) \stackrel{\text { puff out }}{\longrightarrow}\left\{\begin{array}{l}
\mathrm{NS} 5(6789 \psi)+\mathrm{P}(\psi) \\
\mathrm{NS} 5(6789 \mathcal{C})+\mathrm{P}(\mathcal{C})
\end{array}\right.
$$

Lifting this to M-theory along $x^{10}$, this becomes

$$
\mathrm{M} 2(67)+\mathrm{M} 2(89) \stackrel{\text { puff out }}{\longrightarrow}\left\{\begin{array}{l}
\mathrm{M} 5(6789 \psi)+\mathrm{P}(\psi) \\
\mathrm{M} 5(6789 \mathcal{C})+\mathrm{P}(\mathcal{C})
\end{array}\right.
$$


Renaming coordinates as $(6,7,8,9, y, 10) \rightarrow(4,5,6,7,8,9)$, we have

$$
\mathrm{M} 2(45)+\mathrm{M} 2(67) \stackrel{\text { puff out }}{\longrightarrow}\left\{\begin{array}{l}
\mathrm{M} 5(4567 \psi)+\mathrm{P}(\psi) \\
\mathrm{M} 5(4567 \mathcal{C})+\mathrm{P}(\mathcal{C})
\end{array}\right.
$$

This is a supertube transition in the M-theory frame of (2.1). Compactifying M-theory to IIA on $\psi$, this reduces to

$$
\mathrm{D} 2(45)+\mathrm{D} 2(67) \stackrel{\text { puff out }}{\longrightarrow}\left\{\begin{array}{l}
\mathrm{D} 4(4567)+\mathrm{D} 0 \\
\mathrm{NS} 5(4567 \mathcal{C})+\mathrm{P}(\mathcal{C})
\end{array}\right.
$$

This is the process (2.35) in the IIA frame of (2.10).

\section{B Coordinate systems}

Here we summarize relations between the coordinate systems that we use in the main text.

The flat $\mathbb{R}^{4}$ coordinates $x_{m}, m=1,2,3,4$, are variously written as

$$
\begin{aligned}
& x_{1}+i x_{2}=s e^{i \widetilde{\phi}}=2 \sqrt{r} \sin \frac{\theta}{2} e^{i \frac{\psi}{2}}, \\
& x_{3}+i x_{4}=w e^{i \widetilde{\psi}}=2 \sqrt{r} \cos \frac{\theta}{2} e^{i\left(\frac{\psi}{2}+\phi\right)} .
\end{aligned}
$$

The flat $\mathbb{R}^{4}$ metric can be written as

$$
\begin{aligned}
d s_{4}^{2} & =d x_{m} d x_{m} \\
& =d s^{2}+s^{2} d \widetilde{\phi}^{2}+d w^{2}+w^{2} d \widetilde{\psi}^{2} \\
& =\frac{1}{r} d r^{2}+r\left(d \theta^{2}+2(1-\cos \theta) d \widetilde{\phi}^{2}+2(1+\cos \theta) d \widetilde{\psi}^{2}\right) \\
& =V^{-1}(d \psi+\mathcal{A})^{2}+V d \mathbf{y}^{2}, \\
\mathcal{A} & =(1+\cos \theta) d \phi, \quad V=\frac{1}{r}, \quad *_{3} d \mathcal{A}=d V,
\end{aligned}
$$

where

$$
\begin{aligned}
d \mathbf{y}^{2} & =d r^{2}+r^{2}\left(d \theta^{2}+\sin ^{2} \theta d \phi^{2}\right), \\
y_{1}+i y_{2} & =r \sin \theta e^{i \phi}, \quad y_{3}=r \cos \theta .
\end{aligned}
$$

In the toroidal coordinates

$$
y_{1}+i y_{2}=\frac{\sqrt{u^{2}-1}}{u-\cos \sigma} R e^{i \phi}, \quad y_{3}^{\prime} \equiv y_{3}-c=\frac{\sin \sigma}{u-\cos \sigma} R,
$$

the flat $\mathbb{R}^{3}$ metric can be written as

$$
d \mathbf{y}^{2}=d \mathbf{y}^{\prime 2}=d y_{1}^{2}+d y_{2}^{2}+d y_{3}^{\prime 2}=\frac{R^{2}}{(u-\cos \sigma)^{2}}\left[\frac{d u^{2}}{u^{2}-1}+d \sigma^{2}+\left(u^{2}-1\right) d \phi^{2}\right]
$$


where $\mathbf{y}^{\prime}=\left(y_{1}, y_{2}, y_{3}^{\prime}\right)$. The inverse relations are

$$
\cos \sigma=\frac{\mathbf{y}^{\prime 2}-R^{2}}{\Lambda}, \quad u=\frac{\mathbf{y}^{\prime 2}+R^{2}}{\Lambda}, \quad \Lambda^{2}=\left(\mathbf{y}^{\prime 2}-R^{2}\right)^{2}+4 R^{2} y_{3}^{\prime 2} .
$$

Some relations between different coordinates:

$$
\begin{aligned}
r & =\frac{s^{2}+w^{2}}{4}=\frac{1}{4}|\vec{x}|^{2}, \quad \sin \frac{\theta}{2}=\frac{s}{\sqrt{s^{2}+w^{2}}}, \quad \cos \frac{\theta}{2}=\frac{w}{\sqrt{s^{2}+w^{2}}} \\
\cos \theta & =\frac{-s^{2}+w^{2}}{s^{2}+w^{2}}, \quad \sin \theta=\frac{2 s w}{s^{2}+w^{2}} \\
s & =2 \sqrt{r} \sin \frac{\theta}{2}=\sqrt{2 r(1-\cos \theta)}=\sqrt{2\left(r-y_{3}\right)}, \\
w & =2 \sqrt{r} \cos \frac{\theta}{2}=\sqrt{2 r(1+\cos \theta)}=\sqrt{2\left(r+y_{3}\right)} \\
\rho & =\sqrt{y_{1}^{2}+y_{2}^{2}}=r \sin \theta=\frac{s w}{2}, \quad y_{3}=r \cos \theta=\frac{-s^{2}+w^{2}}{4} .
\end{aligned}
$$

The relation between the position of the ring in $\mathbb{R}^{4}$ (specified by $a, b$ ) and in $\mathbb{R}^{3}$ (specified by $R, c)$ :

$$
R=\frac{a b}{2}, \quad c=\frac{-a^{2}+b^{2}}{4}, \quad \sqrt{R^{2}+c^{2}}=\frac{a^{2}+b^{2}}{4} .
$$

Some more relations:

$$
\begin{aligned}
& V=\frac{1}{r}=\frac{4}{s^{2}+w^{2}}=\frac{4}{|\vec{x}|^{2}} \\
& \Lambda^{2}=\frac{1}{256}\left[(s+a)^{2}+(w+b)^{2}\right]\left[(s+a)^{2}+(w-b)^{2}\right] \\
& \times\left[(s-a)^{2}+(w+b)^{2}\right]\left[(s-a)^{2}+(w-b)^{2}\right] \\
& u=\frac{\left(s^{2}+w^{2}\right)^{2}+\left(a^{2}+b^{2}\right)^{2}-2\left(a^{2}-b^{2}\right)\left(s^{2}-w^{2}\right)}{\sqrt{\left[(s+a)^{2}+(w+b)^{2}\right]\left[(s+a)^{2}+(w-b)^{2}\right]\left[(s-a)^{2}+(w+b)^{2}\right]\left[(s-a)^{2}+(w-b)^{2}\right]}}, \\
& \zeta=\sqrt{1-u^{-2}}=\frac{8 a b s w}{\left(s^{2}+w^{2}\right)^{2}+\left(a^{2}+b^{2}\right)^{2}-2\left(a^{2}-b^{2}\right)\left(s^{2}-w^{2}\right)}, \\
& \Sigma=\frac{1}{4} \sqrt{\left[(s+a)^{2}+w^{2}\right]\left[(s-a)^{2}+w^{2}\right]}=|\mathbf{y}-\widetilde{\mathbf{a}}|, \\
& \widetilde{\mathbf{a}}=(0,0,-\widetilde{a}), \quad \widetilde{a}=\frac{a^{2}}{4}, \quad \widetilde{b}=\frac{b^{2}}{4}
\end{aligned}
$$

\section{Functions $\boldsymbol{H}, \mathcal{I}_{m n}, \gamma$}

Here we summarize the functions introduced in the main text and discuss their properties.

\section{C.1 The harmonic function $H$}

The harmonic function $H$ can be written in various ways as

$$
\begin{aligned}
H & =\frac{1}{R} \sqrt{\frac{u-\cos \sigma}{2 u}}{ }_{2} F_{1}\left(\frac{1}{4}, \frac{3}{4} ; 1 ; \zeta^{2}\right)=\frac{2}{\pi R} \sqrt{\frac{u-\cos \sigma}{2 u}} \frac{\mathbf{K}\left(\frac{2 \zeta}{\zeta-1}\right)}{\sqrt{1-\zeta}} \\
& =\frac{1}{R} \sqrt{\frac{u-\cos \sigma}{2}} P_{-1 / 2}(u)=\frac{4 \mathcal{I}_{00}(P, Q)}{s^{2}+a^{2}+w^{2}+b^{2}} .
\end{aligned}
$$


where $\zeta=\sqrt{1-u^{-2}}$ and $\mathbf{K}(m)$ is the complete elliptic integral of the first kind. The value of $H$ at $r=0$ is easy to see from the last expression, because $r=0$ means $P=Q=0$ :

$$
H(r=0)=\frac{4}{a^{2}+b^{2}}
$$

The behavior of $H$ near the ring and near infinity is given in (3.13).

$H$ appears in the functions $Z_{1,2}$ of the Lunin-Mathur geometry for the helical profile (3.1) after smearing. For example, $Z_{2}$ is

$$
\begin{aligned}
Z_{2} & =\frac{Q_{5}}{L} \int_{0}^{L} \frac{d \lambda}{|\vec{x}-\vec{g}|^{2}} \\
& =\frac{Q_{5}}{L} \int_{0}^{2 \pi} \frac{d \lambda}{s^{2}+a^{2}+w^{2}+b^{2}-2 s a \cos (\widetilde{\phi}-k \Omega \lambda)-2 w b \cos \left(\widetilde{\psi}+k^{\prime} \Omega \lambda\right)} .
\end{aligned}
$$

After smearing along $\psi$, this becomes

$$
\begin{aligned}
Z_{2} & =Q_{5} \int_{0}^{2 \pi} \frac{d \mu}{2 \pi} \int_{0}^{2 \pi} \frac{d \mu}{2 \pi} \frac{1}{s^{2}+a^{2}+w^{2}+b^{2}-2 s a \cos \mu-2 w b \cos \nu} \\
& =\frac{Q_{5}}{s^{2}+a^{2}+w^{2}+b^{2}} \int_{0}^{2 \pi} \frac{d \mu}{2 \pi} \int_{0}^{2 \pi} \frac{d \nu}{2 \pi} \frac{1}{1-P \cos \mu-Q \cos \nu} \\
& =\frac{Q_{5}}{s^{2}+a^{2}+w^{2}+b^{2}} \mathcal{I}_{00}(P, Q)=\frac{Q_{5}}{4} H .
\end{aligned}
$$

We can relate this to an integral over a $3 \mathrm{~d}$ profile. Let us set $\nu \rightarrow \nu+\mu$ in the second expression of (C.4) so that

$$
\begin{gathered}
P \cos \mu+Q \cos \nu \rightarrow P \cos \mu+Q \cos (\nu+\mu)=S \cos \left(\mu+\mu_{0}\right) \\
S^{2}=P^{2}+Q^{2}+2 P Q \cos \nu, \quad \cos \mu_{0}=\frac{P+Q \cos \nu}{S}, \quad \sin \mu_{0}=\frac{Q \sin \nu}{S} .
\end{gathered}
$$

If we use this and carry out the $\mu$ integral, we find

$$
\begin{aligned}
Z_{2} & =\frac{Q_{5}}{s^{2}+a^{2}+w^{2}+b^{2}} \int_{0}^{2 \pi} \frac{d \nu}{2 \pi} \frac{1}{\sqrt{1-S^{2}}} \\
& =\frac{Q_{5}}{s^{2}+a^{2}+w^{2}+b^{2}} \int_{0}^{2 \pi} \frac{d \nu}{2 \pi} \frac{1}{\sqrt{1-\left(P^{2}+Q^{2}\right)-2 P Q \cos \nu}} .
\end{aligned}
$$

We can show that this is equal to the integral over the $3 \mathrm{~d}$ profile:

$$
Q_{5} \int_{0}^{2 \pi} \frac{d \nu}{2 \pi} \frac{1}{|\mathbf{y}-\mathbf{f}|}=\frac{Q_{5}}{4} \int \frac{d \nu}{2 \pi} \frac{1}{\sqrt{\rho^{2}+R^{2}-2 \rho R \cos \nu+\left(y_{3}-c\right)^{2}}}
$$

where $\mathbf{f}$ is given by (3.18), because, from (B.8e) and (B.9), it follows that

$$
\rho^{2}+R^{2}-2 \rho R \cos \nu+\left(y_{3}-c\right)^{2}=\frac{\left(s^{2}+w^{2}+a^{2}+b^{2}\right)^{2}}{16}\left[1-\left(P^{2}+Q^{2}\right)-2 P Q \cos \nu\right] .
$$

This means that $H$ can be written as an integral over a $4 \mathrm{~d}$ or $3 \mathrm{~d}$ profile as

$$
H=\left.\frac{4}{L} \int_{0}^{L} \frac{d \lambda}{|\vec{x}-\vec{g}|^{2}}\right|_{\text {smear }}=\int_{0}^{2 \pi} \frac{d \nu}{2 \pi} \frac{1}{|\mathbf{y}-\mathbf{f}|}
$$




\section{C.2 The integrals $\mathcal{I}_{m n}$}

$\mathcal{I}_{m n}$ is defined as

$$
\begin{aligned}
\mathcal{I}_{m n}(P, Q) & \equiv \int_{0}^{2 \pi} \frac{d \mu}{2 \pi} \int_{0}^{2 \pi} \frac{d \nu}{2 \pi} \frac{(P \cos \mu)^{m}(Q \cos \nu)^{n}}{1-P \cos \mu-Q \cos \nu} \\
P & \equiv \frac{2 s a}{s^{2}+a^{2}+w^{2}+b^{2}}, \quad Q \equiv \frac{2 w b}{s^{2}+a^{2}+w^{2}+b^{2}} .
\end{aligned}
$$

The value at $r=0$ is easy to find because $r=0$ means $P=Q=0$. Namely,

$$
\mathcal{I}_{m n}(r=0)= \begin{cases}1 & (m=n=0), \\ 0 & \text { (otherwise) }\end{cases}
$$

One can evaluate $\mathcal{I}_{00}$ as follows. First,

$$
\begin{aligned}
\mathcal{I}_{00} & =\int_{0}^{2 \pi} \frac{d \mu}{2 \pi} \int_{0}^{2 \pi} \frac{d \nu}{2 \pi} \frac{1}{1-P \cos \mu-Q \cos \nu}=\int_{0}^{2 \pi} \frac{d \nu}{2 \pi} \frac{1}{\sqrt{(1-Q \cos \nu)^{2}-P^{2}}} \\
& =\oint \frac{d x}{2 \pi i} \frac{1}{\sqrt{\left(x-(Q / 2)\left(x^{2}+1\right)\right)^{2}-P^{2} x^{2}}}, \quad x=e^{i \nu},
\end{aligned}
$$

where the contour for the $x$ integral is the unit circle on the complex $x$ plane. The roots of the quartic polynomial inside the square root are $x=x_{a}, x_{b}, x_{c}, x_{d}$ with

$$
\begin{aligned}
x_{d}=\frac{1+P-\sqrt{(1+P)^{2}-Q^{2}}}{4 Q}, & x_{c} & =\frac{1-P-\sqrt{(1-P)^{2}-Q^{2}}}{4 Q}, \\
x_{b}=\frac{1-P+\sqrt{(1-P)^{2}-Q^{2}}}{4 Q}, & x_{a} & =\frac{1+P+\sqrt{(1+P)^{2}-Q^{2}}}{4 Q},
\end{aligned}
$$

where $0<x_{d}<x_{c}<1<x_{b}<x_{a}$ for $P, Q, P+Q \in(0,1)$. From this it immediately follows that $[59,3.147 .2]$

$$
\mathcal{I}_{00}=\frac{4}{\pi} \frac{\mathbf{K}(m)}{Q \sqrt{\left(x_{a}-x_{c}\right)\left(x_{b}-x_{d}\right)}},
$$

where

$$
\begin{gathered}
m=\frac{\left(x_{a}-x_{b}\right)\left(x_{c}-x_{d}\right)}{\left(x_{a}-x_{c}\right)\left(x_{b}-x_{d}\right)}=\left(\frac{\sqrt{1-(P-Q)^{2}}-\sqrt{1-(P+Q)^{2}}}{\sqrt{1-(P-Q)^{2}}+\sqrt{1-(P+Q)^{2}}}\right)^{2}, \\
\frac{1}{Q \sqrt{\left(x_{a}-x_{c}\right)\left(x_{b}-x_{d}\right)}}=\frac{1}{\sqrt{1-(P+Q)^{2}}+\sqrt{1-(P-Q)^{2}}} .
\end{gathered}
$$

By using the identity [60]

$$
\mathbf{K}(z)=\frac{2}{1+\sqrt{1-z}} \mathbf{K}\left(\left(\frac{1-\sqrt{1-z}}{1+\sqrt{1-z}}\right)^{2}\right)
$$

one can obtain the second expression in (C.1). 
Likewise, we can write $\mathcal{I}_{01}, \mathcal{I}_{10}$ using elliptic integrals. For example,

$$
\begin{aligned}
\mathcal{I}_{01} & =\frac{1}{\pi} \int_{x_{d}}^{x_{c}} d x \frac{x}{\sqrt{\left(x_{a}-x\right)\left(x_{b}-x\right)\left(x_{c}-x\right)\left(x-x_{d}\right)}} \\
& =\frac{4}{\pi} \frac{1}{\sqrt{\left(x_{a}-x_{c}\right)\left(x_{b}-x_{d}\right)}}\left[\left(x_{d}-x_{a}\right) \Pi\left(\frac{x_{d}-x_{c}}{x_{a}-x_{c}} \mid m\right)+x_{a} \mathbf{K}(m)\right]
\end{aligned}
$$

where $\Pi(n \mid m)$ is the complete elliptic integral of the third kind.

The 1-form $\mathbf{A}$ in the Lunin-Mathur geometry can be written in the $(s, \widetilde{\phi}, w, \widetilde{\psi})$ coordinates in terms of $\mathcal{I}_{10}, \mathcal{I}_{01}$ as

$$
\begin{aligned}
& A_{\widetilde{\phi}}=\operatorname{Im}\left[\left(A_{1}+i A_{2}\right) e^{-i \widetilde{\phi}}\right] s=-\frac{Q_{5} \Omega k}{2} \mathcal{I}_{10}, \\
& A_{\widetilde{\psi}}=\operatorname{Im}\left[\left(A_{3}+i A_{4}\right) e^{-i \widetilde{\psi}}\right] w=+\frac{Q_{5} \Omega k^{\prime}}{2} \mathcal{I}_{01} .
\end{aligned}
$$

\section{C.3 The monodromic harmonic function $\gamma$}

The explicit expression for $\gamma$ was given in (3.20).

The value of $\gamma$ on the $y_{3}$ axis, namely $u=1$, can be found by using the explicit expression (3.20) as

$$
\gamma(u=1, \sigma)=\sin ^{2}\left(\frac{\pi}{2}\left\{\frac{\sigma}{2 \pi}\right\}\right)+\left\lfloor\frac{\sigma}{2 \pi}\right\rfloor
$$

where $\lfloor x\rfloor$ is the floor function and $\{x\} \equiv x-\lfloor x\rfloor$ is the fractional part of $x$.

Let us find the value of $\gamma$ at $r=0$. On the $y_{3}$ axis, the relation between $\sigma$ and $y_{3}^{\prime}=y-c$ is $y_{3}^{\prime}=R \cot (\sigma / 2)$, as can be derived from (3.7). Using this, we can show that the value of $\gamma$ at $r=0\left(y_{3}^{\prime}=-c=\left(a^{2}-b^{2}\right) / 4\right)$ is

$$
\gamma(r=0) \equiv \frac{b^{2}}{a^{2}+b^{2}}+n
$$

where $n \in \mathbb{Z}$ must be taken appropriately for the branch in consideration. Let us consider the branch

$$
-\pi<\sigma<\pi, \quad \text { therefore } \quad-\frac{1}{2}<\gamma(u=1, \sigma)<\frac{1}{2} .
$$

With this choice, $\gamma$ vanishes at infinity (which corresponds to $u=1, \sigma=0$ ). In this branch, $\gamma(r=0)$ is

$$
\gamma(r=0)= \begin{cases}\frac{b^{2}}{a^{2}+b^{2}} & (a>b), \\ -\frac{a^{2}}{a^{2}+b^{2}} & (b>a) .\end{cases}
$$




\section{C.4 Various relations}

We can always write $\mathcal{I}_{10}$ and $\mathcal{I}_{01}$ as follows:

$$
\begin{aligned}
& x \mathcal{I}_{10}+y \mathcal{I}_{01}= \int_{0}^{2 \pi} \frac{d \mu}{2 \pi} \int_{0}^{2 \pi} \frac{d \nu}{2 \pi} \frac{x P \cos \mu+y Q \cos \nu}{1-P \cos \mu-Q \cos \nu} \\
&=\int_{0}^{2 \pi} \frac{d \mu}{2 \pi} \int_{0}^{2 \pi} \frac{d \nu}{2 \pi}\left[\frac{x-y}{a^{2}+b^{2}} \frac{b^{2} P \cos \mu-a^{2} Q \cos \nu}{1-P \cos \mu-Q \cos \nu}\right.\left.\quad+\frac{a^{2} x+b^{2} y}{a^{2}+b^{2}} \frac{1-(1-P \cos \mu-Q \cos \nu)}{1-P \cos \mu-Q \cos \nu}\right] \\
&=\frac{x-y}{a^{2}+b^{2}}\left(b^{2} \mathcal{I}_{10}-a^{2} \mathcal{I}_{01}\right)+\frac{a^{2} x+b^{2} y}{a^{2}+b^{2}}\left(\frac{H}{V}+\frac{a^{2}+b^{2}}{4} H-1\right) .
\end{aligned}
$$

Let us derive the near-ring behavior of $\mathcal{I}_{00}$. Near the ring, where $s \rightarrow a, w \rightarrow b$, we have

$$
P \approx \frac{a^{2}}{a^{2}+b^{2}}, \quad Q \approx \frac{b^{2}}{a^{2}+b^{2}}, \quad P+Q \approx 1
$$

So, in the integral

$$
\mathcal{I}_{00}=\int \frac{d \mu}{2 \pi} \frac{1}{\sqrt{(1-P \cos \mu)^{2}-Q^{2}}}
$$

the dominant contribution comes from $\mu \approx 0$, which corresponds to the contribution from the part of the ring near the point where we are sitting. In this limit, $(1-P \cos \mu)^{2}-$ $Q^{2} \approx(1-P+Q)\left(D^{2}+x^{2}\right)$, where $D \equiv \sqrt{1-P-Q}$ is the distance from us to the ring which is now approximated to be straight, and $x=\sqrt{P / 2} \mu$ is the distance along the straight ring. So,

$$
\begin{aligned}
\mathcal{I}_{00} & \approx \frac{1}{2 \pi} \sqrt{\frac{2}{(1-P+Q) P}} \int_{-\Lambda}^{\Lambda} \frac{d x}{\sqrt{D^{2}+x^{2}}} \\
& \approx \frac{1}{\pi} \sqrt{\frac{2}{(1-P+Q) P}} \log \frac{\Lambda}{D}=\frac{1}{\pi} \frac{a^{2}+b^{2}}{a b} \log \frac{\Lambda}{D},
\end{aligned}
$$

where $\Lambda$ is a cutoff which depends on global data and cannot be determined by a local analysis here. The relation between $D$ and $u$ works out to be $D \approx \frac{a b}{\sqrt{2}\left(a^{2}+b^{2}\right)} \frac{1}{u}$. So, in the end

$$
\mathcal{I}_{00} \sim \frac{1}{\pi} \frac{a^{2}+b^{2}}{a b} \log u
$$

Evaluation of $\mathcal{I}_{10}$ is mostly identical, because $\cos \mu \approx 1$ in the $\mu \approx 0$ region. The result is

$$
\mathcal{I}_{10} \sim \frac{a^{2}}{a^{2}+b^{2}} \frac{1}{\pi} \frac{a^{2}+b^{2}}{a b} \log u=\frac{a}{\pi b} \log u, \quad \mathcal{I}_{01} \sim \frac{b}{\pi a} \log u .
$$

This in particular means that

$$
\left.\triangle\left(b^{2} \mathcal{I}_{10}-a^{2} \mathcal{I}_{01}\right)=\text { regular } \quad \text { (no } \delta \text { function }\right) .
$$


Let us briefly discuss how to show that (3.28) is equal to (3.29), namely

$$
b^{2} \mathcal{I}_{10}-a^{2} \mathcal{I}_{01}=\frac{4 a b}{\left(s^{2}+w^{2}+a^{2}+b^{2}\right)^{2}} \int_{0}^{2 \pi} \frac{d \mu}{4 \pi} \frac{\left(b^{2}-a^{2}\right) s w \cos \mu+a b\left(s^{2}-w^{2}\right)}{\sqrt{X}(1+\sqrt{X})},
$$

where $X \equiv 1-\left(P^{2}+Q^{2}\right)-2 P Q \cos \mu$. Just as we did around (C.4), by setting $\nu \rightarrow \nu+\mu$ and carrying out the $\nu$ integral first, we find

$$
\mathcal{I}_{10}=\int_{0}^{2 \pi} \frac{d \mu}{2 \pi} \frac{P(P+Q \cos \mu)}{\sqrt{1-S^{2}}\left(1+\sqrt{1-S^{2}}\right)} .
$$

$\mathcal{I}_{01}$ is obtained by $P \leftrightarrow Q$. Using the relations (B.8e), (B.9), it is straightforward to prove (C.32).

Open Access. This article is distributed under the terms of the Creative Commons Attribution License (CC-BY 4.0), which permits any use, distribution and reproduction in any medium, provided the original author(s) and source are credited.

\section{References}

[1] K. Behrndt, D. Lüst and W.A. Sabra, Stationary solutions of $N=2$ supergravity, Nucl. Phys. B 510 (1998) 264 [hep-th/9705169] [INSPIRE].

[2] J.P. Gauntlett, J.B. Gutowski, C.M. Hull, S. Pakis and H.S. Reall, All supersymmetric solutions of minimal supergravity in five- dimensions, Class. Quant. Grav. 20 (2003) 4587 [hep-th/0209114] [INSPIRE].

[3] B. Bates and F. Denef, Exact solutions for supersymmetric stationary black hole composites, JHEP 11 (2011) 127 [hep-th/0304094] [INSPIRE].

[4] I. Bena and N.P. Warner, One ring to rule them all ... and in the darkness bind them?, Adv. Theor. Math. Phys. 9 (2005) 667 [hep-th/0408106] [INSPIRE].

[5] J.P. Gauntlett and J.B. Gutowski, General concentric black rings, Phys. Rev. D 71 (2005) 045002 [hep-th/0408122] [INSPIRE].

[6] I. Bena and N.P. Warner, Bubbling supertubes and foaming black holes, Phys. Rev. D $\mathbf{7 4}$ (2006) 066001 [hep-th/0505166] [INSPIRE].

[7] P. Meessen and T. Ortín, The supersymmetric configurations of $N=2, D=4$ supergravity coupled to vector supermultiplets, Nucl. Phys. B $\mathbf{7 4 9}$ (2006) 291 [hep-th/0603099] [INSPIRE].

[8] S. Ferrara, R. Kallosh and A. Strominger, $N=2$ extremal black holes, Phys. Rev. D 52 (1995) R5412 [hep-th/9508072] [INSPIRE].

[9] A. Strominger, Macroscopic entropy of $N=2$ extremal black holes, Phys. Lett. B 383 (1996) 39 [hep-th/9602111] [INSPIRE].

[10] S. Ferrara and R. Kallosh, Supersymmetry and attractors, Phys. Rev. D 54 (1996) 1514 [hep-th/9602136] [INSPIRE].

[11] S. Ferrara and R. Kallosh, Universality of supersymmetric attractors, Phys. Rev. D 54 (1996) 1525 [hep-th/9603090] [inSPIRE].

[12] G.W. Moore, Strings and Arithmetic, in Les Houches School of Physics: Frontiers in Number Theory, Physics and Geometry, (2007), pp. 303-359, DOI [hep-th/0401049] [INSPIRE]. 
[13] P. Kraus and F. Larsen, Attractors and black rings, Phys. Rev. D 72 (2005) 024010 [hep-th/0503219] [INSPIRE].

[14] F. Larsen, The Attractor Mechanism in Five Dimensions, Lect. Notes Phys. 755 (2008) 249 [hep-th/0608191] [INSPIRE].

[15] F. Denef, On the correspondence between D-branes and stationary supergravity solutions of type-II Calabi-Yau compactifications, in Workshop on Strings, Duality and Geometry, (2000) [hep-th/0010222] [INSPIRE].

[16] F. Denef, (Dis)assembling special Lagrangians, hep-th/0107152 [INSPIRE].

[17] G.W. Moore, PiTP lectures on BPS states and wall-crossing in $d=4, N=2$ theories, http://www.physics.rutgers.edu/ gmoore/PiTP_July26_2010.pdf.

[18] F. Denef and G.W. Moore, Split states, entropy enigmas, holes and halos, JHEP 11 (2011) 129 [hep-th/0702146] [INSPIRE].

[19] P. Berglund, E.G. Gimon and T.S. Levi, Supergravity microstates for BPS black holes and black rings, JHEP 06 (2006) 007 [hep-th/0505167] [INSPIRE].

[20] P. Heidmann and S. Mondal, The full space of BPS multicenter states with pure D-brane charges, JHEP 06 (2019) 011 [arXiv:1810.10019] [INSPIRE].

[21] D. Mateos and P.K. Townsend, Supertubes, Phys. Rev. Lett. 87 (2001) 011602 [hep-th/0103030] [INSPIRE].

[22] M. Park and M. Shigemori, Codimension-2 solutions in five-dimensional supergravity, JHEP 10 (2015) 011 [arXiv: 1505.05169] [INSPIRE].

[23] J.J. Fernandez-Melgarejo, M. Park and M. Shigemori, Non-Abelian Supertubes, JHEP 12 (2017) 103 [arXiv: 1709.02388] [INSPIRE].

[24] O. Lunin and S.D. Mathur, AdS/CFT duality and the black hole information paradox, Nucl. Phys. B 623 (2002) 342 [hep-th/0109154] [INSPIRE].

[25] O. Lunin, J.M. Maldacena and L. Maoz, Gravity solutions for the D1-D5 system with angular momentum, hep-th/0212210 [INSPIRE].

[26] B.E. Niehoff and N.P. Warner, Doubly-Fluctuating BPS Solutions in Six Dimensions, JHEP 10 (2013) 137 [arXiv: 1303.5449] [INSPIRE].

[27] I. Bena, P. Kraus and N.P. Warner, Black rings in Taub-NUT, Phys. Rev. D 72 (2005) 084019 [hep-th/0504142] [INSPIRE].

[28] R. Gregory, J.A. Harvey and G.W. Moore, Unwinding strings and t duality of Kaluza-Klein and h monopoles, Adv. Theor. Math. Phys. 1 (1997) 283 [hep-th/9708086] [InSPIRE].

[29] D. Marolf, Chern-Simons terms and the three notions of charge, in International Conference on Quantization, Gauge Theory, and Strings: Conference Dedicated to the Memory of ProfeSSOR Efim Fradkin, (2000), pp. 312-320 [hep-th/0006117] [INSPIRE].

[30] A.S. Schwarz, Field theories with no local conservation of the electric charge, Nucl. Phys. B 208 (1982) 141 [INSPIRE].

[31] J.B. Gutowski and H.S. Reall, General supersymmetric AdS $S_{5}$ black holes, JHEP 04 (2004) 048 [hep-th/0401129] [INSPIRE].

[32] J.B. Gutowski and W. Sabra, General supersymmetric solutions of five-dimensional supergravity, JHEP 10 (2005) 039 [hep-th/0505185] [INSPIRE]. 
[33] K. Hanaki, K. Ohashi and Y. Tachikawa, Comments on charges and near-horizon data of black rings, JHEP 12 (2007) 057 [arXiv: 0704.1819] [INSPIRE].

[34] F. Denef, Supergravity flows and D-brane stability, JHEP 08 (2000) 050 [hep-th/0005049] [INSPIRE].

[35] G. Dall'Agata, S. Giusto and C. Ruef, U-duality and non-BPS solutions, JHEP 02 (2011) 074 [arXiv: 1012.4803] [INSPIRE].

[36] A. Strominger and C. Vafa, Microscopic origin of the Bekenstein-Hawking entropy, Phys. Lett. B 379 (1996) 99 [hep-th/9601029] [INSPIRE].

[37] V.S. Rychkov, D1-D5 black hole microstate counting from supergravity, JHEP 01 (2006) 063 [hep-th/0512053] [INSPIRE].

[38] C. Krishnan and A. Raju, A Note on D1-D5 Entropy and Geometric Quantization, JHEP 06 (2015) 054 [arXiv : 1504.04330] [INSPIRE].

[39] I. Kanitscheider, K. Skenderis and M. Taylor, Fuzzballs with internal excitations, JHEP 06 (2007) 056 [arXiv: 0704.0690] [InSPIRE].

[40] S. Giusto and R. Russo, Superdescendants of the D1D5 CFT and their dual 3-charge geometries, JHEP 03 (2014) 007 [arXiv: 1311.5536] [INSPIRE].

[41] I. Bena, N. Bobev, C. Ruef and N.P. Warner, Supertubes in Bubbling Backgrounds: Born-Infeld Meets Supergravity, JHEP 07 (2009) 106 [arXiv:0812.2942] [INSPIRE].

[42] S. Giusto, S. Rawash and D. Turton, Ads 3 holography at dimension two, JHEP 07 (2019) 171 [arXiv: 1904.12880] [INSPIRE].

[43] J. de Boer and M. Shigemori, Exotic Branes in String Theory, Phys. Rept. 532 (2013) 65 [arXiv:1209.6056] [INSPIRE].

[44] A.W. Peet, TASI lectures on black holes in string theory, in Theoretical Advanced Study Institute in Elementary Particle Physics (TASI 99): Strings, Branes, and Gravity, (2000), pp. 353-433, DOI [hep-th/0008241] [INSPIRE].

[45] R. Emparan, D. Mateos and P.K. Townsend, Supergravity supertubes, JHEP 07 (2001) 011 [hep-th/0106012] [INSPIRE].

[46] I. Kanitscheider, K. Skenderis and M. Taylor, Holographic anatomy of fuzzballs, JHEP 04 (2007) 023 [hep-th/0611171] [INSPIRE].

[47] K. Skenderis and M. Taylor, Fuzzball solutions and D1-D5 microstates, Phys. Rev. Lett. 98 (2007) 071601 [hep-th/0609154] [INSPIRE].

[48] J.R. David, G. Mandal and S.R. Wadia, Microscopic formulation of black holes in string theory, Phys. Rept. 369 (2002) 549 [hep-th/0203048] [InSPIRE].

[49] S.G. Avery, Using the D1D5 CFT to Understand Black Holes, arXiv:1012.0072 [INSPIRE].

[50] I. Bena et al., Asymptotically-flat supergravity solutions deep inside the black-hole regime, JHEP 02 (2018) 014 [arXiv:1711.10474] [INSPIRE].

[51] A. Schwimmer and N. Seiberg, Comments on the $N=2, N=3, N=4$ Superconformal Algebras in Two-Dimensions, Phys. Lett. B 184 (1987) 191 [INSPIRE].

[52] I. Bena, N. Bobev and N.P. Warner, Spectral Flow, and the Spectrum of Multi-Center Solutions, Phys. Rev. D 77 (2008) 125025 [arXiv:0803.1203] [InSPIRE]. 
[53] S. Giusto, O. Lunin, S.D. Mathur and D. Turton, D1-D5-P microstates at the cap, JHEP 02 (2013) 050 [arXiv: 1211.0306] [INSPIRE].

[54] S. Hampton, S.D. Mathur and I.G. Zadeh, Lifting of D1-D5-P states, JHEP 01 (2019) 075 [arXiv: 1804.10097] [INSPIRE].

[55] O. Lunin and S.D. Mathur, Rotating deformations of $A d S_{3} \times S^{3}$, the orbifold CFT and strings in the pp wave limit, Nucl. Phys. B 642 (2002) 91 [hep-th/0206107] [INSPIRE].

[56] J. Gomis, L. Motl and A. Strominger, PP wave/CFT(2) duality, JHEP 11 (2002) 016 [hep-th/0206166] [INSPIRE].

[57] E. Gava and K.S. Narain, Proving the PP wave/CFT(2) duality, JHEP 12 (2002) 023 [hep-th/0208081] [INSPIRE].

[58] I. Bena, B.D. Chowdhury, J. de Boer, S. El-Showk and M. Shigemori, Moulting Black Holes, JHEP 03 (2012) 094 [arXiv:1108.0411] [InSPIRE].

[59] I.S. Gradshteyn and I.M. Ryzhik, Table of Integrals, Series and Products, Academic Press (2014).

[60] http://functions.wolfram.com/08.02.17.0003.01. 